DAMTP-2008-80

\title{
Universal properties of the near-horizon optical geometry
}

\author{
G W Gibbons* \& C M Warnick ${ }^{\dagger}$ \\ DAMTP, University of Cambridge, \\ Wilberforce Road, Cambridge \\ CB3 0WA, UK
}

\begin{abstract}
We make use of the fact that the optical geometry near a static non-degenerate Killing horizon is asymptotically hyperbolic to investigate universal features of black hole physics. We show how the Gauss-Bonnet theorem allows certain lensing scenarios to be ruled in or out. We find rates for the loss of scalar, vector and fermionic 'hair' as objects fall quasistatically towards the horizon. In the process we find the Liénard-Wiechert potential for hyperbolic space and calculate the force between electrons mediated by neutrinos, extending the flat space result of Feinberg and Sucher. We use the enhanced conformal symmetry of the Schwarzschild and Reissner-Nordström backgrounds to re-derive the electrostatic field due to a point charge in a simple fashion.
\end{abstract}

\section{Introduction}

There has been over the past few years a very large amount of theoretical work on black holes addressing problems in quantum gravity, supergravity, string theory and M-theory. Typically one seeks solutions of the supergravity equations in four or higher dimensions, and while many are broadly similar to to the well known Kerr-Newman-de-Sitter family in four spacetime dimensions, there are many differences of detail and in higher dimensions qualitatively different features can arise. It is desirable therefore to fix upon universal properties, true for a broad class of black holes. For this reason the near horizon geometry of extreme black holes has received a great deal of attention, since it universally behaves like $A d S_{2} \times M_{n-2}$, where $M_{n-2}$ is typically an $n-2$ dimensional Einstein space and the symmetry is enhanced from $\mathbb{R}$ to $S O(2,1)$. By contrast the universal near horizon optical geometry of non-extreme horizons with its enhanced conformal symmetry has largely been ignored (notable exceptions are [1, 2]). In other words, little has been done to exploit the fact that near a non-extreme horizon of a static black hole with metric

$$
d s^{2}=-V^{2} d t^{2}+\gamma_{i j} d x^{i} d x^{j}
$$

*g.w.gibbons@damtp.cam.ac.uk

${ }^{\dagger}$ c.m.warnick@damtp.cam.ac.uk 
$i=1,2, \ldots, n-1$ the optical metric

$$
a_{i j} d x^{i} d x^{j}=V^{-2} \gamma_{i j} d x^{i} d x^{j},
$$

becomes asymptotically hyperbolic, with a conformal boundary whose geometry is that of the event horizon. In the spherically symmetric case, the limiting optical geometry is precisely that of hyperbolic space $H^{n-1}=S O(n-1,1) / S O(n-1)$ with radius of curvature equal to $\kappa^{-1}$, where $\kappa$ is the surface gravity.

This is especially ironic because asymptotically hyperbolic geometry has been studied for some time because of the light it throws on the no-hair properties of asymptotically de-Sitter metrics and the freezing of perturbations which have crossed the horizon of an inflationary universe (see [3] for a recent discussion and references to earlier work). A much better known case arises in the AdS/CFT correspondence where the asymptotically hyperbolic geometry of $A d S_{n}$ or, in its "Euclidean" formulation, $H^{n}$ is of interest.

The aim of the present paper is to fill this gap by embarking on an exploration of what can be learned about the universal qualitative properties of black holes from studying their near horizon optical geometry using the tools of hyperbolic geometry. We shall principally be concerned with the two topics

- A qualitative study of of null geodesics near a static horizon using the Gauss-Bonnet theorem, rather in the style of [4] in the case of cosmic strings.

- A study of the shedding of 'hair' near static event horizons using propagators in hyperbolic space.

Of course, in the case of astrophysical black holes the near horizon geometry has long been studied under the rubric of the "Membrane Paradigm" [5] and its Rindler like features have been described. However this work, mainly concentrates on the planar approximation to the horizon geometry and does not make use of detailed concepts and ideas of hyperbolic geometry. Closer to what we are interested in is the work of Haba [2] which considers scalar fields near a Killing horizon using an optical geometry approach and constructs approximate Green's functions in cases where the horizon is not necessarily spherical. This approach is more in tune with our philosophy of seeking universal properties. We will focus on spherical horizons and show that the enhanced symmetry present in this case make approximate propagators much simpler to construct. We remark that the universal nature of black hole absorption cross-section [6] has recently played an important rôle in the understanding of the ratio of shear viscosity to entropy density of conformal fluid in the AdS / CFT correspondence [7].

The paper will be organised as follows: we first define the optical metric and explore some of its properties, including a study of light rays near an event horizon using the Gauss-Bonnet theorem. We will then present a general argument based on the near horizon limit of the optical geometry to estimate the rate of loss of 'hair' as bodies fall towards the black hole. Then we will show how the optical metric allows one to find the fields due to static electric and scalar charges in the Schwarzschild and Reissner-Nordström backgrounds with very little calculation. This is a re-derivation of results in the literature in a more coherent and direct way. We will include equipotential plots for a charged particle approaching a black hole, graphically demonstrating the no-hair result. 


\section{Optical Metrics}

The optical metric may be thought of as the modern incarnation of an idea dating back to Fermat in the 17th century. Fermat expressed the laws governing reflection and refraction of light as what we would now call an action principle. His 'principle of least time' states that the path taken by a ray of light is that which minimises the time taken between the two points. This can be used to derive the more familiar Snell's law and other optical laws.

In the case of light rays moving in a static background, with a given choice of time coordinate $t$, we may take this at face value and define the action for light rays in the metric

$$
g=-a^{2}(\boldsymbol{x}) d t^{2}+h_{i j}(\boldsymbol{x}) d x^{i} d x^{j}
$$

to be

$$
S=\int d t=\int \sqrt{a^{-2} h_{i j} \frac{d x^{i}}{d \lambda} \frac{d x^{j}}{d \lambda}} d \lambda,
$$

where we use the fact that null rays have $d s=0$. Extremizing this action gives the unparameterised geodesics of the 3 dimensional Riemannian metric:

$$
h_{\mathrm{opt} .}=a^{-2}(\boldsymbol{x}) h_{i j}(\boldsymbol{x}) d x^{i} d x^{j}
$$

These unparameterised geodesics are the light rays and the metric $h_{\mathrm{opt}}$ is the optical metric. One may check that these unparameterised geodesics indeed coincide with the projections of the null geodesics of (2.1) onto the spacelike surfaces $t=$ const. and so the light rays are the paths traced by photons moving in this static space. The equivalence is clear by considering the metric

$$
g_{\text {opt. }}=a^{-2} g=-d t^{2}+h_{\text {opt. }}
$$

since the unparameterised null geodesics are conformally invariant objects, the result follows. We will sometimes refer to the ultra-static metric $g_{\text {opt. }}$ as the optical metric also, relying on context to distinguish it from $h_{\text {opt. }}$. The optical metric is not necessarily unique as it depends upon a choice of time coordinate $t$. For metrics which admit more than one choice of $t$ there can be more than one optical metric. We shall see this in detail in the case of anti-de Sitter space below.

It is not only statements about the null geodesics which are accessible via the optical metric. Many of the field equations of physics both classical and quantum behave well under conformal transformations and so we can make use of the universal nature of the near horizon optical geometry to study physics near a black hole (or cosmological) horizon.

\subsection{The Optical Metrics of de Sitter and anti-de Sitter}

\subsection{1 de Sitter}

We start with $3+1$ dimensional de Sitter as the timelike hyperboloid in $\mathbb{E}^{4,1}$ :

$$
X^{2}+Y^{2}+Z^{2}+W^{2}-V^{2}=1, \quad d s^{2}=d X^{2}+d Y^{2}+d Z^{2}+d W^{2}-d V^{2} .
$$

We could consider $n+1$ dimensions, but the generalisations are straightforward. A choice of static time coordinate $t$ corresponds to a choice of future-directed, timelike, hypersurface orthogonal 
Killing vector $\frac{\partial}{\partial t}$. The Killing vectors of $\mathrm{dS}$ are those in $\mathbb{E}^{4,1}$ which generate rotations and boosts. A basis for the Killing vectors is given by the hypersurface orthogonal vectors:

$$
M_{\mu \nu}=X_{\mu} \frac{\partial}{\partial X^{\nu}}-X_{\nu} \frac{\partial}{\partial X^{\mu}} .
$$

Here $\mu, \nu$ are $\mathbb{E}^{4,1}$ indices. There is no Killing vector which is everywhere timelike, however the Killing vector:

$$
K=W \frac{\partial}{\partial V}+V \frac{\partial}{\partial W}
$$

is timelike and future directed in the region $\left\{W^{2}-V^{2}>0\right\} \cup\{W>0\}$. Furthermore, any other choice of timelike Killing vector is equivalent to $K$ under a Lorentz transformation. We can find a parameterisation of the hyperboloid in this patch, such that $K=\frac{\partial}{\partial t}$ is a static Killing vector as follows:

$$
\begin{aligned}
X & =r \sin \theta \sin \phi, \\
Y & =r \sin \theta \cos \phi \\
Z & =r \cos \theta \\
W & =\sqrt{1-r^{2}} \cosh t \\
V & =\sqrt{1-r^{2}} \sinh t .
\end{aligned}
$$

On this patch, the metric takes the form

$$
d s^{2}=\left(1-r^{2}\right)\left(-d t^{2}+\frac{d r^{2}}{\left(1-r^{2}\right)^{2}}+\frac{r^{2}}{1-r^{2}}\left(d \theta^{2}+\sin ^{2} \theta d \phi^{2}\right)\right)
$$

so that the optical metric may be seen to be the Beltrami metric on Hyperbolic space. In fact these coordinates cover all of the Beltrami ball and so the optical geometry of the static slicing of de Sitter is precisely $\mathbb{H}^{3}$. The conformal infinity of the hyperbolic ball corresponds to the Killing horizon on the hyperboloid at $W^{2}-V^{2}=0$ where $K$ becomes null. It is a general characteristic of Killing horizons that the optical geometry approaches a constant negative curvature geometry near the horizon.

\subsubsection{Anti-de Sitter}

The situation for AdS is somewhat more interesting than that for dS because there exist three equivalence classes of timelike, future directed, hypersurface orthogonal Killing vectors under the action of $S O(3,2)$. To see this we take AdS to be a hyperboloid in $\mathbb{E}^{3,2}$ :

$$
-W^{2}-V^{2}+X^{2}+Y^{2}+Z^{2}=-1, \quad d s^{2}=-d W^{2}-d V^{2}+d X^{2}+d Y^{2}+d Z^{2} .
$$

In a similar way to the case of $\mathrm{dS}$, a basis for the Killing vectors is given by:

$$
M_{\mu \nu}=X_{\mu} \frac{\partial}{\partial X^{\nu}}-X_{\nu} \frac{\partial}{\partial X^{\mu}},
$$


where $\mu, \nu$ are $\mathbb{E}^{3,2}$ indices. Under a $S O(3,2)$ transformation, any Killing vector which is timelike somewhere on the hyperboloid may be brought into one of three forms, listed below with the region in which they are timelike:

$$
\begin{array}{lll}
K_{1}=V \frac{\partial}{\partial W}-W \frac{\partial}{\partial V}, & & \text { all of AdS, } \\
K_{2}=(Z+W) \frac{\partial}{\partial V}+V\left(\frac{\partial}{\partial Z}-\frac{\partial}{\partial W}\right), & & \{W+Z>0\}, \\
K_{3}=Z \frac{\partial}{\partial V}+V \frac{\partial}{\partial Z}, & & \left\{Z^{2}-V^{2}>0\right\} \cup\{Z>0\} .
\end{array}
$$

We now find the optical metric in each case:

Case 1 We pick the following parameterisation of the hyperboloid

$$
\begin{aligned}
X & =r \sin \theta \sin \phi \\
Y & =r \sin \theta \cos \phi \\
Z & =r \cos \theta \\
W & =\sqrt{1+r^{2}} \cos t \\
V & =\sqrt{1+r^{2}} \sin t,
\end{aligned}
$$

so that the metric is given by:

$$
d s^{2}=\left(1+r^{2}\right)\left(-d t^{2}+\frac{d r^{2}}{\left(1+r^{2}\right)^{2}}+\frac{r^{2}}{1+r^{2}}\left(d \theta^{2}+\sin ^{2} \theta d \phi^{2}\right)\right),
$$

and we recognise that the optical metric is the Beltrami metric for $S^{3}$. This covers one half of the sphere, with the 2 -sphere at $r=\infty$ corresponding to an equatorial 2 -sphere.

Case 2 We pick a different parameterisation for the hyperboloid:

$$
\begin{aligned}
X & =x / z \\
Y & =y / z \\
Z & =\left(1+t^{2}-x^{2}-y^{2}-z^{2}\right) /(2 z), \\
W & =\left(1-t^{2}+x^{2}+y^{2}+z^{2}\right) /(2 z), \\
V & =t / z,
\end{aligned}
$$

so that the metric is the Poincaré upper half-space metric:

$$
d s^{2}=\frac{1}{z^{2}}\left(-d t^{2}+d x^{2}+d y^{2}+d z^{2}\right), \quad z>0
$$

and the optical geometry is the half-space $\{z>0\}$ in $\mathbb{E}^{3}$.

Case 3 Finally we consider the case where $\frac{\partial}{\partial t}=K_{3}$. A suitable parameterisation of the static patch is provided by:

$$
\begin{aligned}
X & =\tan \theta \cos \phi \\
Y & =\tan \theta \sin \phi \\
Z & =\cosh t \operatorname{cosech} \chi \sec \theta \\
W & =\operatorname{cotanh} \chi \sec \theta \\
V & =\sinh t \operatorname{cosech} \chi \sec \theta
\end{aligned}
$$


The spatial coordinates have ranges $0 \leq \phi<2 \pi, 0 \leq \theta<\pi / 2,0<\chi$. The metric is given by:

$$
d s^{2}=\operatorname{cosech}^{2} \chi \sec ^{2} \theta\left(-d t^{2}+d \chi^{2}+\sinh ^{2} \chi\left(d \theta^{2}+\sin ^{2} \theta d \phi^{2}\right)\right) .
$$

We see that the optical metric is that of hyperbolic space in geodesic polar coordinates. Since $\theta$ does not range over $[0, \pi)$ the coordinates only cover half of $\mathbb{H}^{3}$ and there is a boundary which is given by the plane $\theta=\pi / 2$ in these coordinates.

We note that all three of the AdS optical metric have a finite boundary. This boundary corresponds to the conformal infinity of the AdS space and is a manifestation of the fact that $\mathrm{AdS}$ is not globally hyperbolic. In the case of $\mathrm{dS}$, the optical metric is complete and has an asymptotically hyperbolic end which corresponds to the Killing horizon of the static patch. As

we will see below, this behaviour is typical of a Killing horizon, such as the event horizons of Schwarzschild and Reissner-Nordström.

\subsection{The Optical Metric of Schwarzschild and Reissner-Nordström}

Owing to the similarities between the cosmological horizon of de Sitter and the event horizons of black holes, we might expect the optical geometries to be similar near the horizon. We shall see that this is indeed the case, and that near the horizon, the geometry of a static black hole has an asymptotically hyperbolic optical metric.

We start with the Schwarzschild metric

$$
d s^{2}=-\left(1-\frac{2 M}{r}\right) d t^{2}+\frac{d r^{2}}{1-\frac{2 M}{r}}+r^{2}\left(d \theta^{2}+\sin ^{2} \theta d \phi^{2}\right)
$$

and make the coordinate transformation

$$
r=M\left(\frac{1+\rho}{\rho}\right)
$$

this takes the asymptotically flat end to $\rho=0$ and the horizon to $\rho=1$ and puts the metric into the form:

$$
d s^{2}=\left(\frac{1-\rho}{1+\rho}\right)\left[-d t^{2}+16 M^{2}\left(\frac{1+\rho}{2 \rho}\right)^{4}\left\{\frac{d \rho^{2}}{\left(1-\rho^{2}\right)^{2}}+\frac{\rho^{2}}{1-\rho^{2}}\left(d \theta^{2}+\sin ^{2} \theta d \phi^{2}\right)\right\}\right]
$$

The term inside the braces may be seen to be the metric on $\mathbb{H}^{3}$ in Beltrami coordinates. In the limit $\rho \rightarrow 1$, we thus see that the optical metric tends to a metric of constant negative curvature as we approach the horizon.

The case of Reissner-Nordström is rather similar, although the resulting metric is not so elegant. In the familiar coordinates, the Reissner-Nordström metric is given by

$$
d s^{2}=-\left(1-\frac{2 M}{r}+\frac{Q^{2}}{r^{2}}\right) d t^{2}+\frac{d r^{2}}{1-\frac{2 M}{r}+\frac{Q^{2}}{r^{2}}}+r^{2}\left(d \theta^{2}+\sin ^{2} \theta d \phi^{2}\right)
$$


In these coordinates, the horizon is at $r=M+\sqrt{M^{2}-Q^{2}}=M+\mu$, where we define a new parameter $\mu$ which we will assume to be strictly positive. The case $\mu=0$ corresponds to an extremal black hole which we will not consider here. The coordinate transformation

$$
r=M+\frac{\mu}{\rho}
$$

puts the metric into the form

$$
d s^{2}=\frac{\mu^{2}\left(1-\rho^{2}\right)}{(\mu+m \rho)^{2}}\left[-d t^{2}+\frac{(\mu+m)^{4}}{\mu^{2}}\left(\frac{\mu+m \rho}{(\mu+m) \rho}\right)^{4}\left\{\frac{d \rho^{2}}{\left(1-\rho^{2}\right)^{2}}+\frac{\rho^{2}}{1-\rho^{2}}\left(d \theta^{2}+\sin ^{2} \theta d \phi^{2}\right)\right\}\right]
$$

We see once again that the optical metric approaches the Beltrami metric on hyperbolic space as we get close to the horizon. In both cases, the radius of the hyperbolic space is $\beta_{H}$, the inverse Hawking temperature of the black hole. In fact, this is a general property of a static metric with a non-degenerate Killing horizon as shown in [1]. In that paper Sachs and Solodukhin show that near a non-extreme horizon of a static black hole with metric

$$
d s^{2}=-V^{2} d t^{2}+\gamma_{i j} d x^{i} d x^{j},
$$

$i=1,2, \ldots, n-1$ the optical metric

$$
a_{i j} d x^{i} d x^{j}=V^{-2} \gamma_{i j} d x^{i} d x^{j}
$$

becomes asymptotically hyperbolic, with a conformal boundary whose geometry is that of the event horizon. Essentially this is due to the fact that at a non degenerate Killing horizon $V^{2}$ must have a simple zero. We will consider the spherically symmetric case from here on, however we will try and identify results which we expect to remain the same in the case of more interesting (compact) horizon topology.

It will prove crucial in our exact calculations later that the optical metrics of both Schwarzschild and Reissner-Nordström take the form:

$$
g_{\text {opt. }}=-d t^{2}+H^{4} h,
$$

where $h$ is the metric on the unit pseudo-sphere, $\mathbb{H}^{3}$, and $H$ is a harmonic function on $\mathbb{H}^{3}$ which approaches 1 near the conformal boundary of $\mathbb{H}^{3}$. This observation is responsible for the fact that the fields due to static electric and scalar charges in these backgrounds may be found explicitly [8, 9, 10]. We show below how these fields may be constructed. This special form of the metric occurs only in the 4-dimensional space-times, so does not, unfortunately, lead to a generalisation of these results in an obvious way to higher dimensions.

For another viewpoint on the optical geometry of Schwarzschild see [11] where the geometry is constructed as an embedding in a higher dimensional hyperbolic space.

\section{Lensing and The Gauss-Bonnet theorem}

In order to discuss null geodesics we could follow the well trodden path of solving the differential equations. Instead we will follow the approach of [4, 12, and extract information about geodesics 
using the Gauss-Bonnet theorem which directly involves the negative curvature of the optical metric. Although here we consider only the Schwarzschild black hole, it is clear that many qualitative properties may be deduced using only the assumption of negative curvature near the horizon of the optical metric, provided a totally geodesic 2-surface exists.

Let us now consider geodesics lying in an oriented two-surface $\Sigma$. We may apply the GaussBonnet theorem to obtain useful information [4], in particular about angle sums of geodesic triangles. Let $D \subset \Sigma$ be domain with Euler number $\chi(D)$ and a not necessarily connected boundary $\partial D$, possibly with corners at which the tangent vector of the boundary is discontinuous. If $K$ is the Gauss curvature of $D$, such that $R_{i j k l}=K\left(f_{i k} f_{j l}-f_{i l} f_{j k}\right)$ and $k$ the curvature of $\partial D, \theta_{i}$ the angle through which the tangent turns inwards at the i'th corner then

$$
\int_{D} K d A+\oint_{\partial D} k d l+\sum_{i} \theta_{i}=2 \pi \chi(D) .
$$

In the case of the Schwarzschild metric, if one considers geodesics in an equatorial plane the optical metric is

$$
d s^{2}=\frac{d r^{2}}{\left(1-\frac{2 M}{r}\right)^{2}}+\frac{r^{2}}{\left(1-\frac{2 M}{r}\right)} d \phi^{2} .
$$

We return here to the standard Schwarzschild coordinates of (2.19). Note that the radial optical distance is

$$
\frac{d r}{\left(1-\frac{2 M}{r}\right)}=d r^{\star}
$$

where $r^{\star}=r-2 M+2 M \ln \left(\frac{r}{2 M}-1\right)$ is the Regge-Wheeler tortoise coordinate.

There is a circular geodesic at $r=3 M$ and the horizon $r=2 M$ is at an infinite optical distance inside this at $r^{\star}=-\infty$. The Gauss curvature

$$
K=-\frac{2 M}{r^{3}}\left(1-\frac{3 M}{2 r}\right)
$$

is everywhere negative. It falls to zero like $-\frac{2 M}{r^{3}}$ at infinity but near the horizon the Gauss-curvature approaches the negative constant $-\frac{1}{(4 M)^{2}}$. This is precisely as we expect to find given the results of the previous section.

The fact that the Gauss curvature is negative looks on the face of it rather paradoxical, since one usually thinks of gravitational fields as focussing a bundle of light rays. However, as Lodge perhaps dimly realised [13] a spherical vacuum gravitational field does not quite act in that way. The equation of geodesic deviation governing the separation $\eta$ of two neighbouring light rays in the equatorial plane is

$$
\frac{d^{2} \eta}{d t^{2}}+K \eta=0
$$

Thus neighbouring light rays actually diverge. The focussing effect of a gravitational lens is not, as we shall see shortly, a local but rather a global, indeed even topological, effect.

One might wonder whether the full 3-dimensional curvature ${ }^{3} R_{i j k l}$ of the optical metric has all of its sectional curvatures negative, but this cannot be. The sectional curvature of a surface is related to the full curvature tensor by

$$
{ }^{3} R_{i j k l}=K\left(f_{i k} f_{j l}-f_{1 l} f_{j k}\right)-K_{i k} K_{j l}+K_{i l} g_{j k},
$$



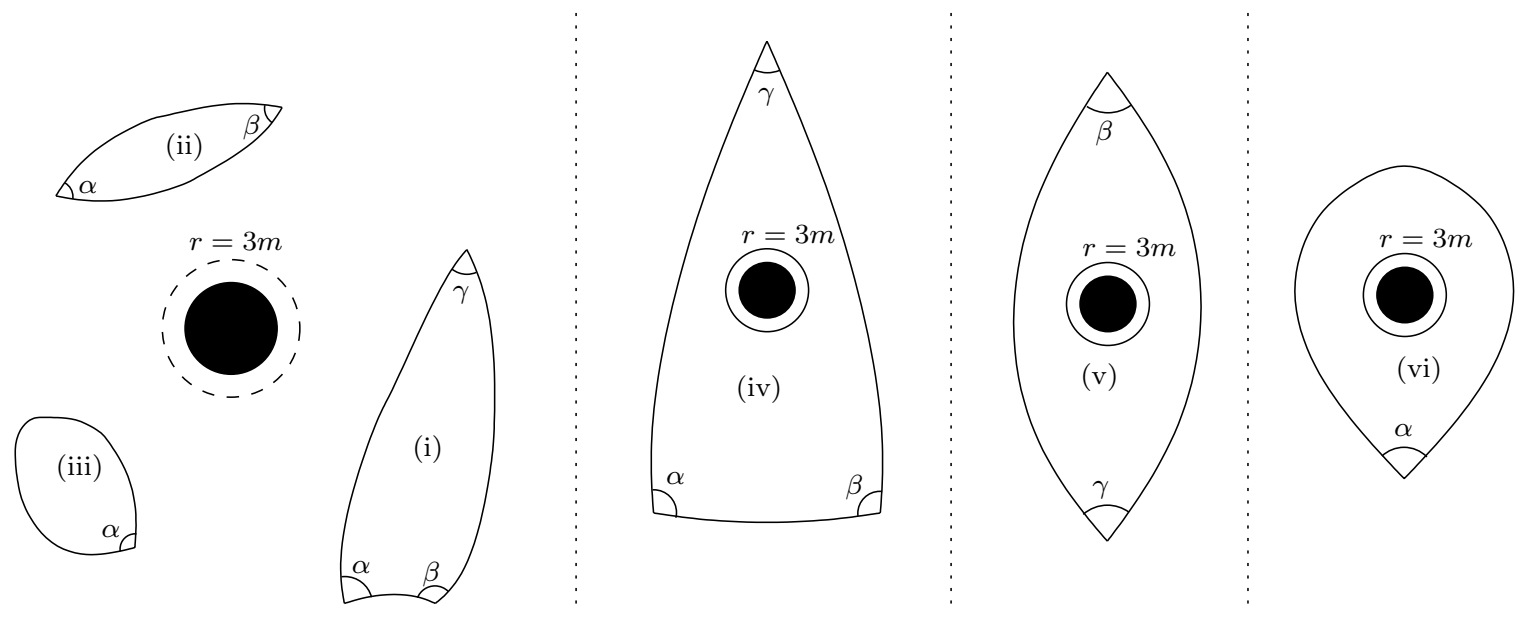

Figure 1: The geodesic polygons described in (i)-(vi)

where $K_{i j}$ is the second fundamental form or extrinsic curvature of the surface. For a totally geodesic surface $K_{i j}=0$, and the two sectional curvatures agree. One such totally geodesic surface is the equatorial plane for which, as we have seen, $K$ is negative. Another totally geodesic submanifold is the sphere at $r=3 M$ for which $K$ is obviously positive.

The negativity of the Gauss curvature of the optical metric in the equatorial plane is a fairly universal property of black hole metrics. To see this we note that if

$$
d s^{2}=d \rho^{2}+l^{2}(\rho) d \phi^{2}
$$

then

$$
K=-\frac{1}{l} \frac{d^{2} l}{d \rho^{2}} .
$$

Any metric with the same qualitative features as the Schwarzschild metric, as long as it has a positive mass, will have $K$ negative. Indeed this fact might be made the basis of excluding negative mass objects observationally.

A simple calculation shows the integral over the region outside the circular geodesic at $r=3 M$ is

$$
\int_{r \geq 3 M} K d A=-2 \pi .
$$

Let us now apply the Gauss-Bonnet theorem to various cases.

(i) Geodesic triangle $\Delta$ not containing the the region inside $r=3 M$. In this case $\chi(\Delta)=1$. If $\alpha, \beta, \gamma$ are the necessarily positive internal angles, we find that the angle sum is less that $\pi$,

$$
\alpha+\beta+\gamma=\pi+\int_{\Delta} K d A<\pi .
$$

(ii) Geodesic di-gon $S$ not containing the the region inside $r=3 M$. In this case $\chi(S)=1$. If $\alpha$ and $\beta$ are the internal angles,

$$
\alpha+\beta=\int_{S} K d A<0
$$


In other words two such geodesics cannot intersect twice if the hole is not inside the di-gon. Neither, in these circumstances, can a geodesic intersect itself because

(iii) Geodesic loop $T$ not containing the the region inside $r=3 M$. In this case $\chi(T)=1$ and one finds that if if $\alpha$ is the internal angles, the

$$
\alpha=-\pi+\int_{T} K d A<-\pi
$$

which is plainly impossible.

This might seem counter-intuitive in the light of one's usual intuition about light bending, but this feeling is dispelled by considering cases in which the domain $D$ has two boundary components, the second, inner, one being the circular geodesic at $r=3 \mathrm{M}$. The domain with the circle removed has the topology of an annulus and thus its Euler number vanishes.

(iv) Geodesic triangle with hole $\Delta_{o}$ enclosing the geodesic circle at $r=3 M$ and with the region the region inside $r=3 M$ removed.

If $\alpha, \beta, \gamma$ are the internal angles, we find that the angle sum is greater than $\pi$,

$$
\alpha+\beta+\gamma=3 \pi+\int_{\Delta_{0}} K d A \geq \pi
$$

Similarly

(v) Geodesic di-gon $S_{0}$ with the the region inside $r=3 M$ removed. In this case $\chi\left(S_{0}\right)=0$ and one finds that if If $\alpha$ and $\beta$ are the internal angles, then

$$
\alpha+\beta=2 \pi+\int_{S_{0}} K d A>0 .
$$

In other words two such geodesics may intersect twice if the hole is inside the di-gon. Moreover, in these circumstances, a geodesic can intersect itself because:

(vi) Geodesic loop $T_{0}$ containing the the region inside $r=3 M$. In this case $\chi\left(T_{0}\right)=1$ and if If $\alpha$ is the internal angle, we find that

$$
\alpha=\pi+\int_{T_{0}} K d A
$$

which is plainly possible.

Similar results may be obtained by considering geodesics inside $r=3 M$, but now domain must not contain the horizon, otherwise $\int_{D} K d A$ will diverge. Near the horizon the geometry is that of Lobachevsky space with constant curvature $-\frac{1}{4 M}$.

(vii) Deflection. We consider a geodesic line with no self-intersection which at large distances, is radial. The angle between the asymptotes is $\delta$, with the convention that it is positive if the light ray is bent towards the hole. The geodesic decomposes the region inside two circles, one of very large radius and the other at $r=3 M$ into two domains $D_{ \pm}$whose common boundary component consists of the geodesic, which intersects the circle at infinity at right angles. We chose $D_{+}$to enclose the hole so it has an inner boundary component at $r=3 M$ and a portion of the circle at infinity through which the angle $\phi$ has range $\pi-\delta$. Clearly $D_{+}$is topologically an annulus and so 


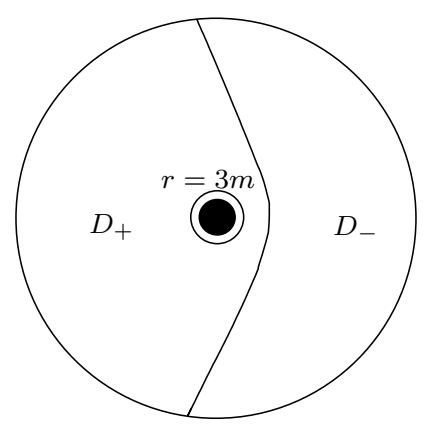

Figure 2: Light bending by a Schwarzschild black-hole

it has vanishing Euler number, $\chi\left(D_{+}\right)=0$. The other domain has Euler number $\chi\left(D_{-}\right)=1$, and $\phi$ ranges through $\pi+\delta$. The Gauss-Bonnet formula applied to $D_{ \pm}$acquires a contribution from the two corners and the circle at infinity. The result is

$$
\delta=-\int_{D_{-}} K d A>0 .
$$

For a geodesic whose distance of closest approach is very large, we may estimate this integral by approximating the geodesic as the straight line $r=\frac{b}{\sin \phi}$. The impact parameter to this lowest non-trivial order coincides with the distance of nearest approach and equals $b$. To the necessary accuracy

$$
K d A \approx-\frac{2 M}{r^{3}} r d r d \phi
$$

The domain of integration $D_{-}$is, with sufficient accuracy over $r \geq \frac{b}{\sin \phi}, 0 \leq \phi \leq 2 \pi$. A simple calculation gives the classic result

$$
\delta=\frac{4 M}{b}
$$

Note the same method works for any static metric, not just Schwarzschild (for an application to gravitational lensing see [12]) and shows that the Gauss-Bonnet method does not just give qualitative results, but it can be made into a quantitative tool.

\section{No-hair properties from the optical metric}

We now shift our attention to a different aspect of black hole physics, the so called 'no-hair' property. A stationary black hole has only three measurable quantities associated with it: mass, angular momentum and electric charge. Thus no matter how complicated a system we start with, once it has undergone gravitational collapse to form a black hole, we are left with only these three pieces of information. This presents only a minor philosophical problem if we are prepared to accept that the information contained in the initial system is somehow trapped irretrievably behind the horizon. Once one includes Hawking's observation that black holes may radiate and indeed evaporate over time, the question of where the information goes becomes more vexed, giving rise to the so called 'information loss paradox'. 
We shall be interested with discovering how, at the classical level, information is lost as a body falls into a black hole. We will work with an approximation where the in-falling body is supposed to have a negligible effect on the background and so we may consider physics in a fixed black-hole geometry. This amounts to a linearisation of the problem, but allows analytic progress to be made.

At the linearised level, the no-hair property may be translated mathematically into the notion that the black hole exterior cannot support any external fields which are regular both at the horizon and spacelike infinity. An interesting question is what happens to the fields around some compact body as it falls into the black hole. This corresponds to asking what happens to a propagator as its pole approaches the horizon. This gives information both about the classical scenario, but also about the outcome of scattering experiments performed as the body falls towards the hole [14].

We are interested in finding a fairly general approach to study how 'hair' is lost as bodies carrying charges fall into a black hole. We will argue that this is a property of the geometry close to the black hole horizon, and so we can consider the problem in this region where the geometry simplifies. We translate the question of finding propagators to a problem in the optical metric and then show how we can estimate the rate of information loss in this geometry. As we noted above, near the horizon this geometry approaches that of hyperbolic space irrespective of the details of the black hole under consideration.

\subsection{Physics in $\mathbb{R}_{t} \times \mathbb{H}^{3}$}

There have been investigations of physics in spaces of constant negative curvature for some time and with varying motivations. Callan and Wilczek initiated a study of quantum mechanics on $\mathbb{H}^{4}$ in [15] in order to geometrically regulate the infra-red divergences of Euclidean field theory. In [16] Atiyah and Sutcliffe considered Skyrmions in $\mathbb{H}^{3}$ as a means of finding approximate Skyrmions in $\mathbb{E}^{3}$ for the case where the pion mass is non-zero. Field theories on $\mathbb{H}^{3}$ are also thermodynamically interesting as one might expect, anticipating the Hawking radiation of horizons. A study of some thermodynamic properties, especially Bose-Einstein condensation is shown in [17]. There have also been studies of electrostatics and magnetostatics in hyperbolic space, with particular reference to the Gauss linking formula [18].

Although the references above provide a reasonably comprehensive discussion of physics in $\mathbb{R}_{t} \times \mathbb{H}^{3}$, in the interests of a self-contained exposition we will discuss some aspects here. Using the near horizon limit of the optical metric, this corresponds after a conformal transformation to physics in the neighbourhood of a non-extremal black hole horizon. The fact that analytic progress is possible may be traced to the fact that the metric is conformally equivalent to the inside of the future light-cone of the origin in Minkowski space as follows.

Throughout this section we represent a point in $\mathbb{H}^{3}$ as a point on the unit pseudosphere $\mathbb{H}^{3}=$ $\left\{X \cdot X=-1, X_{0}>0\right\}$ in $\mathbb{E}^{3,1}$. This makes the equations manifestly $S O(3,1)$ invariant and easy to translate between different coordinate systems. The point $(t, X)$ in $\mathbb{R}_{t} \times \mathbb{H}^{3}$ is mapped to a point on the interior of the future light cone of the origin in $\mathbb{E}^{3,1}$ according to:

$$
\begin{aligned}
\phi: \mathbb{R}_{t} \times \mathbb{H}^{3} & \rightarrow\left\{x \in \mathbb{E}^{3,1}, x \cdot x=-1, x_{0}>0\right\} \\
(t, X) & \mapsto x=X e^{t} .
\end{aligned}
$$

If $g$ is the metric on $\mathbb{R}_{t} \times \mathbb{H}^{3}$ and $\eta$ is the standard metric on $\mathbb{E}^{3,1}$, then one finds that:

$$
\phi_{*} \eta=e^{2 t} g
$$


so we have exhibited the conformal equivalence of these two spaces. This means that given any conformally invariant equation whose propagator may be found in Minkowski space, one may find the propagator for $\mathbb{R}_{t} \times \mathbb{H}^{3}$.

\subsubsection{Massless Wave Equation}

Although the massless wave equation is conformally invariant, and so the propagator may be constructed from the known flat space propagator, it is more convenient to directly solve in this case. We seek to solve the equation:

$$
\left(\square_{g}-\frac{1}{6} R_{g}\right) G(t, X ; \tau, Y)=\left(-\partial_{t}^{2}+\Delta_{h}+1\right) G=\delta_{g}^{(4)}((t, X),(\tau, Y)) .
$$

One might think that the appearance of the curvature term above gives rise to an effective mass, however it is important to include this term in the massless wave equation to ensure, for example, that disturbances propagate along the light-cone as one would expect. Following standard treatments, one Fourier transforms in time and takes $\tau=0$ without loss of generality. We then need to solve the Helmholtz equation

$$
\left(\Delta_{h}+1+k^{2}\right) \tilde{G}=\delta_{h}(X, Y)
$$

This has the general solution, found by using geodesic polar coordinates on $\mathbb{H}^{3}$ :

$$
\tilde{G}(X, Y)=\frac{A e^{i k \chi}+B e^{-i k \chi}}{4 \pi \sinh \chi}
$$

where $\chi=D(X, Y)$ and $A+B=1$. The fact that this (and other Green's functions on $\mathbb{H}^{3}$ ) depends only on $D(X, Y)$ is due to the 2-point homogeneity of the space. Undoing the Fourier transform one finds:

$$
G(t, X ; \tau, Y)=A \frac{\delta(t-\tau-D(X, Y))}{\sinh D(X, Y)}+B \frac{\delta(t-\tau+D(X, Y))}{\sinh D(X, Y)}
$$

The choices for $A$ and $B$ determine what combination of the advanced and retarded propagator we have. Note that this propagator is periodic with period $2 \pi i$ in the time coordinate.

\subsubsection{Liénard-Wiechert Potential}

The Liénard-Wiechert Potential describes the electromagnetic field due to a charge $q$ moving in Minkowski space along some path $r(s) \in \mathbb{E}^{3,1}$ where $s$ is any parameter. The potential at a point $x$ is constructed as follows: first find a solution $s_{r}$ to the equation:

$$
(x-r(s)) \cdot(x-r(s))=0
$$

which should correspond to the intersection of the path of the charge with the past light cone for a retarded propagator. The Maxwell field is then determined by the one-form

$$
A=\left.\frac{q}{4 \pi \epsilon_{0}} \frac{\dot{r} \cdot d x}{(x-r) \cdot \dot{r}}\right|_{s=s_{r}}
$$


Note that this is invariant under re-parameterisations of the path of the particle $r(s)$. In calculating $F=d A$ one should be wary since $A$ depends on $x$ both explicitly and also implicitly through $s_{r}$. Differentiating (4.7) one finds that:

$$
d s_{r}=-\frac{(x-r) \cdot d x}{(x-r) \cdot \dot{r}} .
$$

The standard calculations may now be performed and the Maxwell field calculated.

In order to find the field due to a point charge $q$ moving along the curve $(s, R(s)) \in \mathbb{R}_{t} \times \mathbb{H}^{3}$ we will use the conformal invariance of the Maxwell equations. Using the conformal map $\phi$ we may pull back the Liénard-Wiechert potential from Minkowski space. One finds that the lightcone condition may be re-written:

$$
t-s_{r}=D\left(X, R\left(s_{r}\right)\right)
$$

and the potential is given by:

$$
A=\left.\frac{q}{4 \pi \epsilon_{0}} \frac{1}{\dot{R} \cdot X-\sqrt{(R \cdot X)^{2}-1}}[(R \cdot X+\dot{R} \cdot X) d t+R \cdot d X+\dot{R} \cdot d X]\right|_{s=s_{r}} .
$$

Once again the dependence on $(X, t)$ is subtle, but one may calculate the Maxwell field by using:

$$
d s_{r}=\frac{\sqrt{(X \cdot R)^{2}-1} d t+R \cdot d X}{\sqrt{(X \cdot R)^{2}-1}-\dot{R} \cdot X},
$$

which follows from differentiating (4.10).

Calculating the field strength in the limit where the source charge is at a large distance from the observer but $\dot{R} \cdot \dot{R}$ and $\ddot{R} \cdot \ddot{R}$ remain bounded, we find that the field decays like $e^{-\chi}$ with $\chi$ the separation of charge and observer.

We may interpret this in terms of black hole optical geometry which approaches $\mathbb{H}^{3} \times \mathbb{R}_{t}$ near the horizon. In this case, as we shall see later one must add a static spherically symmetric field to enforce the condition that the black hole is uncharged. We have shown that even including the corrections to the electromagnetic field due to the motion of the charge, the field due to a particle falling into a black hole tends to a monopole charge as the particle approaches the horizon.

\subsubsection{Spinors on hyperbolic space}

Having dealt with spin 0 and spin 1 fields on $\mathbb{R}_{t} \times \mathbb{H}^{3}$, the logical next step is to discuss spinors and the Dirac operator on this space. Following Dirac [19], we will write the Dirac equation in terms of objects in the embedding space, $\mathbb{E}^{3,1}$ as this will allow us to maintain $S O(3,1)$ covariance.

We will make use of the following observation: the Dirac algebra for $\mathbb{E}^{3,1}$ may be represented in the form:

$$
\begin{aligned}
& \gamma^{0}=i \sigma_{3} \otimes I_{2}, \\
& \gamma^{i}=\sigma_{2} \otimes \sigma_{i},
\end{aligned}
$$

where $\sigma_{i}$ are the Pauli matrices which form a 2-component representation of the Dirac algebra for $\mathbb{E}^{3}$. We could, if we so chose, construct this 2-component representation by considering Weyl 
spinors, $\chi^{\alpha}$ on an auxiliary $\mathbb{E}^{3,1}$ restricted to a constant time hyperplane, $\Sigma$. These 2-spinors would then transform under Poincaré transformations of this auxiliary $\mathbb{E}^{3,1}$ fixing the hyperplane and would have a natural $L^{2}$ inner product respecting these transformations given by

$$
\left(\chi_{1}, \chi_{2}\right)=\int_{\Sigma} \mu_{\Sigma} \bar{\chi}_{1} \bar{\sigma} \cdot T \chi_{2}=\int_{\Sigma} \mu_{\Sigma} \bar{\chi}_{1} \chi_{2}
$$

We use the notation $\sigma^{\mu}=\left(I_{2}, \sigma_{i}\right), \bar{\sigma}^{\mu}=\left(-I_{2}, \sigma_{i}\right)$. A Dirac spinor on the original $\mathbb{E}^{3,1}$ space is then the product of two 2-component spinors, the first transforming under $S O(1,1)$ which generates the boosts of the Lorentz group and the second under the rotational $S O(3)$. In fact the second spinors transform under the whole $E(3)$ symmetry of $\mathbb{E}^{3}$ but the translations act by the identity. The reason we take this somewhat circuitous approach to constructing the Dirac spinors is that it will allow us to construct spinors for $\mathbb{R}_{t} \times \mathbb{H}^{3}$ respecting the $S O(3,1)$ invariance of $\mathbb{H}^{3}$.

We will now make use of the fact that the metric in the forward light cone of the origin of Minkowski space may be written in the form

$$
d s^{2}=-d t^{2}+t^{2} h
$$

with $h$ the metric on $\mathbb{H}^{3}$. This is sometimes referred to as the Milne universe. This Minkowski space will play the role of the auxiliary space above. Using the standard approach to construct the Dirac operator from the spin connection, one finds that acting on Weyl spinors,

$$
\not D=\bar{\sigma}^{0}\left(\frac{\partial}{\partial t}+\frac{3}{2 t}\right)+\frac{1}{t} \not D^{(h)}
$$

Now any vector in the forward light cone of the origin may be written

$$
W=t X, \quad \text { with } \quad X \cdot X=-1, \quad t>0, \quad X^{0}>0
$$

we may re-write the Dirac operator as

$$
\not D=\bar{\sigma} \cdot \frac{\partial}{\partial W}=\bar{\sigma} \cdot X\left(X \cdot \frac{\partial}{\partial W}+\frac{3}{2} \frac{1}{|W|}\right)+\frac{1}{|W|}\left(|W| \bar{\sigma} \cdot \frac{\partial}{\partial W}-\bar{\sigma} \cdot X X \cdot \frac{\partial}{\partial W}-\frac{3}{2} \bar{\sigma} \cdot X\right),
$$

comparing this with (4.16) we conclude that

$$
\begin{aligned}
\not D^{(h)} & =\bar{\sigma} \cdot \nabla-\bar{\sigma} \cdot X X \cdot \nabla-\frac{3}{2} \bar{\sigma} \cdot X \\
& =-\bar{\sigma} \cdot X\left(\frac{1}{2} \sigma^{\mu} \bar{\sigma}^{\nu}\left(X_{\mu} \nabla_{\nu}-X_{\nu} \nabla_{\mu}\right)-\frac{3}{2}\right) .
\end{aligned}
$$

It may be checked that $\bar{\sigma} \cdot X$ anti-commutes with the RHS of this expression, so it is in fact convenient to take

$$
\begin{aligned}
\not D^{(h)} & =\frac{1}{2} \sigma^{\mu} \bar{\sigma}^{\nu}\left(X_{\mu} \nabla_{\nu}-X_{\nu} \nabla_{\mu}\right)-\frac{3}{2} \\
& =M-\frac{3}{2}
\end{aligned}
$$


the operator $M$ is that introduced by Dirac in [19]. The relation to the standard construction for Dirac operators in a curved space is developed in [20] Following the discussion above, there is a natural $L^{2}$ inner product on the space of Weyl spinors on $\mathbb{H}^{3}$ given by

$$
\left(\chi_{1}, \chi_{2}\right)_{\mathbb{H}^{3}}=\int_{\mathbb{H}^{3}} \mu[X] \overline{\chi_{1}} \bar{\sigma} \cdot X \chi_{2}
$$

Here $\mu[X]$ is the Riemannian volume form of $\mathbb{H}^{3}$. This inner product is positive definite, because $X \cdot X=-1$ and $X^{0}>0$. Importantly, it is also $S O(3,1)$ invariant by construction and so respects all of the symmetries of hyperbolic space.

We can exhibit a set of plane wave eigenfunctions of the Dirac operator by considering the analogous plane wave eigenfunctions for the Laplace operator on $\mathbb{H}^{3}$ exhibited by Moschella and Schaeffer [21]. Let $\chi$ be a constant 2-spinor (in the sense that $\nabla_{\mu} \chi=0$ ), then the spinor

$$
\kappa_{\omega \chi}(X)=\frac{\omega}{(2 \pi)^{\frac{3}{2}}}(\bar{\chi} \bar{\sigma} \cdot X \chi)^{-\frac{3}{2}-i \omega} \chi
$$

satisfies

$$
\not D^{(h)} \kappa_{\omega \chi}(X)=i \omega \kappa_{\omega \chi}(X)
$$

Furthermore these functions tend pointwise to the standard plane wave basis for eigenfunctions on $\mathbb{E}^{3}$ as the radius of curvature of $\mathbb{H}^{3}$ tends to infinity. Obviously $\chi$ and $\lambda \chi$ define the same function, up to scale, for any $\lambda \in \mathbb{C}^{*}$ so that the space of eigenfunctions is $\mathbb{R}_{+} \times \mathbb{C P}^{1}$. Using the results of Moschella and Schaeffer it should be possible to establish the following normalisation and completeness results

$$
\left(\kappa_{\omega \chi}, \kappa_{\omega^{\prime} \chi^{\prime}}\right)_{\mathbb{H}^{3}}=\delta\left(\omega-\omega^{\prime}\right) \delta\left(\epsilon_{\alpha \beta} \chi^{\alpha} \chi^{\prime \beta}\right)
$$

and

$$
\int_{\mathbb{C P}^{1}} \mu[\chi] \int_{0}^{\infty} d \omega \kappa_{\omega \chi}(X) \overline{\kappa_{\omega \chi}}\left(X^{\prime}\right) \bar{\sigma} \cdot X^{\prime}=\delta_{\mathbb{H}^{3}}\left(X, X^{\prime}\right) I_{2}
$$

however this has so far proved difficult. We shall proceed therefore on the assumption that this is the case. For a discussion of integration over $\mathbb{C P}^{1}$ and the measure $\mu[\chi]$, see the appendix. One may at least show that the second result is true as the hyperbolic radius tends to infinity.

We can now construct Dirac spinors on $\mathbb{R}_{t} \times \mathbb{H}^{3}$ by taking the tensor product of a Weyl spinor on $\mathbb{H}^{3}$ with an $S O(1,1)$ spinor. The Dirac operator is given by:

$$
\not D=i \sigma_{3} \otimes I_{2} \frac{\partial}{\partial t}+\sigma_{2} \otimes\left(M-\frac{3}{2}\right) .
$$

The Dirac conjugate is given by

$$
\overline{\psi \otimes \chi}=\bar{\psi} i \sigma_{3} \otimes \bar{\chi} \bar{\sigma} \cdot X
$$

we note that $\gamma^{0}=i \sigma_{3} \otimes I_{2}$ so the Dirac conjugate does not take its standard form. This is related to the fact that we chose to make the Dirac operator on $\mathbb{H}^{3}$ more symmetric by multiplying by $\sigma \cdot X$. We note finally that we may identify

$$
\gamma^{5}=\sigma_{1} \otimes I_{2}
$$


as the chirality matrix which satisfies

$$
\left\{\gamma^{5}, \not D\right\}=0 \quad \text { and } \quad\left(\gamma^{5}\right)^{2}=-I_{4}
$$

We may finally construct a complete set of plane wave solutions to the Dirac equation

$$
\not D \Psi=0
$$

as follows:

$$
\Psi_{s \omega z \lambda}^{0}(X, t)=\frac{\omega}{(2 \pi)^{\frac{3}{2}}} e^{-s i \omega t} \psi_{\lambda} \otimes\left(\bar{\chi}_{z} \bar{\sigma} \cdot X \chi_{z}\right)^{-\frac{3}{2}+s \lambda i \omega} \chi_{z}
$$

where

$$
\psi_{\lambda}=\frac{1}{\sqrt{2}}\left(\begin{array}{l}
1 \\
\lambda
\end{array}\right), \quad \text { and } \quad \chi_{z}=\frac{1}{\sqrt{1+|z|^{2}}}\left(\begin{array}{l}
1 \\
z
\end{array}\right) .
$$

We have introduced the quantum numbers $s= \pm$ which distinguishes the positive and negative energy solutions, $z \in \mathbb{C}$ which parameterizes $\mathbb{C P}^{1}$ under the usual stereographic projection and $\lambda= \pm$, the chirality. With this choice of parameterisation of $\mathbb{C P}^{1}$ the appropriate measure in the completeness relation (4.25) is

$$
\mu[\chi]=\frac{2 i d z d \bar{z}}{\left(1+|z|^{2}\right)^{2}}
$$

which we recognise as the measure on $S^{2}$ under stereographic projection.

We will now use these results to calculate the force between electrons (or indeed other leptons) mediated by neutrino exchange.

\subsubsection{Neutrino mediated forces}

In flat space there is a long range lepton-lepton force mediated by the exchange of a pair of neutrinos. The potential, as shown by Feinberg and Sucher [22], is

$$
V(r)=\frac{G_{W}^{2}}{4 \pi^{3} r^{5}}
$$

where $G_{W}$ is the weak-interaction coupling constant. Their calculation was based on calculating the one-loop scattering of one electron by another mediated by a $\nu \bar{\nu}$ pair. A simpler means of finding this potential, as described by Hartle [23], is to treat the neutrino field as quantum mechanical and the electrons as classical both in their role as a source for the neutrino field and as particles acted on by that field. Hartle shows that in this limit, the neutrino field obeys the modified Dirac equation

$$
\left(i \not D-\frac{G_{W}}{\sqrt{2}} \gamma \cdot N\left(1+\gamma^{5}\right)\right) \Psi(x)=0
$$

where $N_{\mu}$ is the classical electron number current. The equation of motion of an electron in the classical limit is

$$
m \frac{D u^{\mu}}{d \tau}=\frac{G_{W}}{\sqrt{2}} u^{\nu}\left(\partial^{\mu} B_{\nu}-\partial_{\nu} B^{\mu}\right)
$$


where $u^{\mu}$ is the electron's four-velocity and the potential $B^{\mu}$ is given in terms of the neutrino field by:

$$
B^{\mu}(x)=\left\langle\bar{\Psi}(x) \gamma^{\mu}\left(1+\gamma^{5}\right) \Psi(x)\right\rangle-\left\langle\bar{\Psi}^{0}(x) \gamma^{\mu}\left(1+\gamma^{5}\right) \Psi^{0}(x)\right\rangle .
$$

$\Psi(x)$ is the neutrino field with the weak interactions turned on and $\Psi^{0}(x)$ is the same field with the interactions turned off. Both expectation values are taken in vacuum with no free neutrinos. The normalisation of the neutrino field is fixed by the canonical anti-commutation relations which relate the anti-commutators of fields on a spacelike hypersurface $\sigma$. The only non-vanishing bracket is

$$
\left\{\Psi(x), \bar{\Psi}\left(x^{\prime}\right) \gamma \cdot T\right\}=\delta_{\sigma}\left(x, x^{\prime}\right)
$$

where $T$ is the future directed unit normal to $\sigma$. Note that the standard relation would be between $\Psi$ and $\Psi^{\dagger}$, however as noted above, we have chosen to make the Dirac operator simpler at the expense of taking a non-standard Dirac conjugate. For flat space sliced along constant $t$ hyperplanes, (4.38) reduces to the standard relation.

Let us suppose that there is a complete set of solutions to the modified Dirac equation (4.35) with the same quantum numbers as for the source free Dirac equation, so that we may write

$$
\Psi_{s \omega z \lambda}(t, X)=e^{-s i \omega t} \psi_{\lambda} \otimes \kappa_{s \omega z \lambda}(X)
$$

and we will assume the completeness relation

$$
\sum_{s} \int_{\mathbb{C}} \frac{2 i d z d \bar{z}}{\left(1+|z|^{2}\right)^{2}} \int_{0}^{\infty} d \omega \kappa_{s \omega z \lambda}(X) \bar{\kappa}_{s \omega z \lambda}\left(X^{\prime}\right) \bar{\sigma} \cdot X^{\prime}=\delta_{\mathbb{H}^{3}}\left(X, X^{\prime}\right) I_{2}
$$

(note that we don't sum over $\lambda$ here). We may therefore expand the neutrino field in the form

$$
\Psi(t, X)=\sum_{\lambda} \int_{\mathbb{C}} \frac{2 i d z d \bar{z}}{\left(1+|z|^{2}\right)^{2}} \int_{0}^{\infty} d \omega\left\{e^{-i \omega t} \psi_{\lambda} \otimes \kappa_{+\omega z \lambda} b_{\omega z \lambda}+e^{i \omega t} \psi_{\lambda} \otimes \kappa_{-\omega z \lambda} d_{\omega z \lambda}^{\dagger}\right\}
$$

The canonical anti-commutation relations for the neutrino field imply the following non-vanishing relations for the creation operators $b$ and $d$

$$
\left\{b_{\omega z \lambda}, b_{\omega^{\prime} z^{\prime} \lambda^{\prime}}^{\dagger}\right\}=\left\{d_{\omega z \lambda}, d_{\omega^{\prime} z^{\prime} \lambda^{\prime}}^{\dagger}\right\}=\frac{\left(1+|z|^{2}\right)^{2}}{2 i} \delta\left(z-z^{\prime}\right) \delta\left(\omega-\omega^{\prime}\right) \delta_{\lambda \lambda^{\prime}}
$$

while all other brackets vanish. We suppose the existence of a vacuum state $|0\rangle$ such that $b_{\omega z \lambda}|0\rangle=$ $d_{\omega z \lambda}|0\rangle=0$. Using the anti-commutation relations we find that the electric part of the neutrino mediated vector potential, $V=B^{0}$ takes the form

$$
\begin{aligned}
B^{0}(X)=-2 \int_{\mathbb{C}} & \frac{2 i d z d \bar{z}}{\left(1+|z|^{2}\right)^{2}} \int_{0}^{\infty} d \omega\left\{\bar{\kappa}_{-\omega z+}(X) \bar{\sigma} \cdot X \kappa_{-\omega z+}(X)\right. \\
& \left.-\bar{\kappa}_{-\omega z+}^{0}(X) \bar{\sigma} \cdot X \kappa_{-\omega z+}^{0}(X)\right\} .
\end{aligned}
$$

The fact that the coupling has a $1+\gamma^{5}$ factor ensures that only positive chirality modes contribute. 
As we are only interested in effects at the lowest order in $G_{W}$, we may consider an expansion of the spinors $\kappa$ in terms of $G_{W}$. Expanding to first order

$$
\kappa_{s \omega z \lambda}=\kappa_{s \omega z \lambda}^{0}+G_{W} \kappa_{s \omega z \lambda}^{1}
$$

we find that provided we assume that $N_{t}=\delta_{\mathbb{H}^{3}}\left(X, X^{\prime}\right)$ is the only non-zero component of the electron current, the modified Dirac equation (4.35) implies that

$$
\begin{aligned}
& \left(M-\frac{3}{2}+i s \lambda \omega\right) \kappa_{s \omega z \lambda}^{0}=0 \\
& \left(M-\frac{3}{2}+i s \lambda \omega\right) \kappa_{s \omega z \lambda}^{1}=i \sqrt{2} \delta_{\mathbb{H}^{3}}\left(X, X^{\prime}\right) \kappa_{s \omega z \lambda}^{0} .
\end{aligned}
$$

We know from the previous section that the normalised zero'th order spinors take the form

$$
\kappa_{s \omega z \lambda}^{0}=\frac{\omega}{(2 \pi)^{\frac{3}{2}}}\left(\bar{\chi}_{z} \bar{\sigma} \cdot X \chi_{z}\right)^{-\frac{3}{2}+i s \lambda \omega} \chi_{z}
$$

In order to solve the second equation we note that

$$
M^{2}-2 M=\nabla_{\mathbb{H}^{3}}^{2}
$$

where the Laplacian here is the scalar Laplacian acting componentwise to the right. This may be verified by following the argument of Dirac [19] with the appropriate signature and dimension. We see that

$$
\left(M-\frac{3}{2}+i s \lambda \omega\right)\left(M-\frac{1}{2}-i s \lambda \omega\right)=\left[\nabla_{\mathbb{H}^{3}}^{2}+1+\left(\omega+\frac{i s \lambda}{2}\right)^{2}\right] I_{2}
$$

we recognise the right hand side of the equation as the conformal wave equation with a complex wavenumber, for which we have already found the Green's function. Thus we may solve the second of equations (4.45) by

$$
\kappa_{s \omega z \lambda}^{1}(X)=\left[\left(M-\frac{1}{2}-i s \lambda \omega\right) \phi\left(X, X^{\prime}\right)\right] \kappa_{s \omega z \lambda}^{0}\left(X^{\prime}\right)
$$

where

$$
\phi\left(X, X^{\prime}\right)=\frac{i \sqrt{2}}{4 \pi} e^{i s \omega \chi} \frac{e^{-\lambda \chi / 2}}{\sinh \chi}, \quad \text { with } \quad \cosh \chi=-X \cdot X^{\prime} .
$$

There is a choice of sign here corresponding to picking the retarded propagator. We note that after Fourier transforming back to the time domain the propagator will be anti-periodic in time with period $2 \pi i$. We also note that there appears to be a breaking of the symmetry one might expect under $\lambda \rightarrow-\lambda$. This chiral symmetry breaking is a subtle consequence of the negative curvature and is discussed in [15, 24.

Putting this all together, we find

$$
\frac{B^{0}(X)}{G_{W}}=-4 \operatorname{Re} \int_{\mathbb{C}} \frac{2 i d z d \bar{z}}{\left(1+|z|^{2}\right)^{2}} \int_{0}^{\infty} d \omega \bar{\kappa}_{-\omega z+}^{0}(X) \bar{\sigma} \cdot X\left(M-\frac{1}{2}+i \omega\right) \phi(\chi) \kappa_{-\omega z+}^{0}\left(X^{\prime}\right) .
$$


Inserting (4.46) for $\kappa^{0}$, the integrand reduces to the form

$$
\begin{aligned}
& \frac{\omega^{2}}{(2 \pi)^{3}}\left[\left(\bar{\chi}_{z} \bar{\sigma} \cdot X \chi_{z}\right)^{-\frac{3}{2}+i \omega}\left(\bar{\chi}_{z} \bar{\sigma} \cdot X^{\prime} \chi_{z}\right)^{-\frac{1}{2}-i \omega} \frac{\phi^{\prime}(\chi)}{\sinh \chi}\right. \\
& \left.\quad+\left(\bar{\chi}_{z} \bar{\sigma} \cdot X \chi_{z}\right)^{-\frac{1}{2}+i \omega}\left(\bar{\chi}_{z} \bar{\sigma} \cdot X^{\prime} \chi_{z}\right)^{-\frac{3}{2}-i \omega}\left(\left(i \omega-\frac{1}{2}\right) \phi(\chi)-\operatorname{coth} \chi \phi^{\prime}(\chi)\right)\right] .
\end{aligned}
$$

We will first perform the integrals over $\mathbb{C P}^{1}$ which are of the form:

$$
I_{a}=\int_{\mathbb{C}} \frac{2 i d z d \bar{z}}{\left(1+|z|^{2}\right)^{2}}\left(\bar{\chi}_{z} \bar{\sigma} \cdot X \chi_{z}\right)^{-a}\left(\bar{\chi}_{z} \bar{\sigma} \cdot X^{\prime} \chi_{z}\right)^{a-2}
$$

One may verify that

$$
\left(\bar{\chi}_{z} \bar{\sigma}^{\mu} \chi_{z}\right)=\left(1,-\boldsymbol{n}_{z}\right)
$$

where $\boldsymbol{n}_{z}$ is the pull back of $z$ to the unit sphere in $\mathbb{R}^{3}$ under the standard stereographic projection map. $I_{a}$ is Lorentz invariant (see Appendix), so we may assume without loss of generality that

$$
\begin{aligned}
X & =(\cosh \chi, 0,0,-\sinh \chi), \\
X^{\prime} & =(1,0,0,0),
\end{aligned}
$$

and we may integrate over $S^{2}$ using standard spherical polar coordinates so that

$$
I_{a}=\int \frac{\sin \theta d \theta d \phi}{(\cosh \chi+\cos \theta \sinh \chi)^{a}}=\frac{4 \pi \sinh (1-a) \chi}{(1-a) \sinh \chi} .
$$

Note that this is symmetric under $a \rightarrow 2-a$ as it must be since we could have chosen $X$ and $X^{\prime}$ the other way around. Integrating (4.52) over $\mathbb{C P}^{1}$ then, we have after some simplification

$$
\frac{i \sqrt{2} \omega^{2}}{8 \pi^{3}}\left(\frac{1}{\sinh ^{2} \chi}+\frac{2}{\left(1+4 \omega^{2}\right) \sinh ^{4} \chi}\right)+\frac{\sqrt{2} \omega^{2}}{8 \pi^{3}\left(1+4 \omega^{2}\right)} \frac{e^{-2 i \chi \omega}}{\sinh ^{4} \chi}(2 \omega \sinh \chi-i \cosh \chi),
$$

so that

$$
\frac{B^{0}(\chi)}{G_{W}}=-\frac{4 \sqrt{2}}{4 \pi^{3}} \operatorname{Re} \int_{0}^{\infty} d \omega\left\{\frac{\omega^{2}}{1+4 \omega^{2}} \frac{e^{-2 i \chi \omega}}{\sinh ^{4} \chi}(2 \omega \sinh \chi-i \cosh \chi)\right\} .
$$

This integral is manifestly divergent for large $\omega$, however the potential we are interested in is a low energy effect, so we may introduce a large momentum cut-off by sending $\chi \rightarrow \chi-i \epsilon$ which will make the integral converge for large $\omega$ and taking the $\epsilon \rightarrow 0$ limit after calculating the integral. Doing this, we find that the integral may be performed exactly and we find that the neutrino field gives rise to an effective potential:

$$
V(\chi)=\frac{G_{W}}{\sqrt{2}} B^{t}(\chi)=\frac{G_{W}^{2}}{8 \pi^{3} \chi^{2} \sinh ^{4} \chi}\left(\chi \cosh \chi+\sinh \chi-\chi^{2} \text { Shi } \chi\right),
$$

where Shi is the sinh integral:

$$
\text { Shi } \chi=\int_{0}^{\chi} \frac{\sinh t}{t} d t
$$


This equation is valid for any $\chi$ large with respect to the length scale defined by $G_{W}$. We may take the limit where the hyperbolic radius of the space tends to infinity and we find that

$$
V(\chi) \sim \frac{G_{W}^{2}}{4 \pi^{3} \chi^{5}}
$$

which is the result of Feinberg and Sucher for flat space.

One might be concerned by the fact that there appears to be an asymmetry between the right and left handed neutrinos implicit in (4.50) however it is possible to perform the same calculation under the assumption that left handed neutrinos couple to electrons and the answer found is precisely the same.

\subsection{Thermodynamics}

We noted above that the scalar propagator was periodic and the fermion propagator anti-periodic in imaginary time. Reinserting dimensions, the period is given by $2 \pi i R$ where $R$ is the radius of the hyperbolic space. One expects that thermal propagators for a field at temperature $T$ should have an imaginary period equal to $1 / T$, the inverse of the temperature. As we remarked above, for the near horizon optical geometry of a horizon with surface gravity $\kappa, R=\kappa^{-1}$ so we find that fields in the neighbourhood of a horizon are thermalized at a temperature

$$
T_{H}=\frac{\kappa}{2 \pi},
$$

precisely the Hawking temperature of the horizon. Notice that we nowhere had to 'Euclideanize' the time direction of the manifold in order to derive this result.

\subsection{Approximate calculations near the horizon}

Let us consider trying to construct the propagator for some physical field in a black hole background in the limit where the pole of the propagator approaches the horizon. We assume that since we are considering a small perturbation to the background that the equations are linear and moreover that they may be converted by a conformal transformation to equations with respect to the optical metric. Further, since the black-hole background is assumed to be static, we may Fourier transform

with respect to $t$ so that the equations may be expressed in terms of the optical geometry of $t=$ const. slices. We divide these slices into two regions:

- Region I is a region surrounding the horizon, such that in this region the optical metric has constant negative curvature to order $\epsilon$.

- Region II is the complement of region I and contains the asymptotically flat end.

Region I may be thought of as the exterior of a ball in $\mathbb{H}^{3}$ in the case where the horizon has spherical topology. One would expect that if the topology differs for that of the sphere, then this will not have an effect on the propagator in the limit where the pole approaches the horizon, so we assume that Region I is indeed of this form. The conformal infinity of the hyperbolic space corresponds to the horizon of the black hole. We wish to solve the problem:

$$
\mathcal{L} \phi(x)=\mathcal{L}_{h_{\text {opt. }}} \phi(x)+\mathcal{L}^{\prime} \phi(x)=\delta\left(x, x_{0}\right),
$$


where $x_{0}$ is close to the horizon and we have split the linear operator $\mathcal{L}$ into a geometric operator constructed from $h_{\text {opt }}$. and another part $\mathcal{L}^{\prime}$ which is assumed to be small in Region I. This may require shrinking Region I. We do not assume here that $\phi$ is a scalar - the same considerations will apply for fields of any spin.

In region $\mathrm{I}$, the problem simplifies to finding a propagator in hyperbolic space. This is a simplification because $\mathbb{H}^{3}$ is maximally symmetric so one may make use of isometries to move the pole of the propagator around. We define $G_{I}\left(x, x_{0}\right)$ to satisfy the equation on hyperbolic space:

$$
\mathcal{L}_{h} G_{I}\left(x, x_{0}\right)=\delta\left(x, x_{0}\right)
$$

subject to suitable boundary conditions as $x$ approaches conformal infinity (i.e. the horizon).

In region II, we are solving the homogeneous problem

$$
\mathcal{L}_{h_{\text {opt. }}} G_{I I}\left(x, x_{0}\right)+\mathcal{L}^{\prime} G_{I I}\left(x, x_{0}\right)=0
$$

such that $G_{I I}\left(x, x_{0}\right)$ agrees with $G_{I}\left(x, x_{0}\right)$ at the boundary between regions I and II and decays suitably as $x$ approaches the asymptotically flat infinity. The approximate propagator we construct is then given by:

$$
G\left(x, x_{0}\right)=\left\{\begin{array}{ll}
G_{I}\left(x, x_{0}\right)+K(x) & \text { if } x \text { in Region I } \\
G_{I I}\left(x, X_{0}\right)+K(x) & \text { if } x \text { in Region II }
\end{array},\right.
$$

$K$ here is any solution of the homogeneous problem on the whole of the exterior of the black hole which satisfies appropriate boundary conditions both at spacelike infinity and at the horizon. It is these solutions which carry any 'hair' which the black hole may have. The charges carried by the black hole as a result of $K$ do not follow from regularity at infinity or the horizon but must be determined by, for example, integral conservation laws.

We are now ready to describe the limit as the pole of the propagator approaches the horizon. In Region I we see this as the pole of a propagator in $\mathbb{H}^{3}$ moving towards conformal infinity. As the boundary of Region I is a fixed compact surface in $\mathbb{H}^{3}$, the fields on the boundary decay. Typically this decay is exponentially quickly in the hyperbolic distance of the pole from some fixed point. Thus $G_{I I}$ will also decay at this rate, by linearity. Thus only $K$ can remain in the limit as the pole approaches the horizon, with all other terms decaying. If the black hole cannot support a regular external field $K$, then the fields must all approach zero as the pole of the propagator approaches the horizon. In the case of spherical symmetry it is useful to take the boundary of Region I to be a sphere as it its then possible to decompose all the functions into spherical harmonics and the decay rates for each multipole moment can be calculated separately.

We thus have a method to calculate the rates of decay of propagators as their poles approach the horizon in terms of the distance in the optical metric. We may relate the optical distance along a radial geodesic starting from some fixed point, $\chi$, to the proper distance to the horizon along that geodesic in the physical metric, $\delta$, by:

$$
\delta \sim C e^{-\chi}
$$

in the region near the horizon. This allows us to re-express the decay rates in terms of proper distance to the horizon in the physical metric. We will give constructions below for some simple propagators in hyperbolic space which are useful when constructing the approximate propagators in region $\mathrm{I}$. 


\subsubsection{Example - Massive scalar field}

In the case of a massive scalar field satisfying the Klein-Gordon equation, we wish to find a propagator which satisfies:

$$
\square_{g} \psi-m^{2} \psi=\delta\left(x, x_{0}\right) .
$$

Using conformal transformations defined in section (4.4.2), solving this is equivalent to solving the equation

$$
\frac{1}{H^{5}}\left(\Delta_{h} \Phi+\Phi\right)+\frac{1}{H}\left(k^{2}-m^{2} \Omega^{2}\right) \Phi=\frac{1}{H^{6}} \delta_{h}\left(x, x_{0}\right),
$$

where $h$ refers, as always, to the metric on $\mathbb{H}^{3}$ and $H \rightarrow 1, \Omega \rightarrow 0$ as we approach the horizon. This is of the form supposed above and the exterior supports no solutions to the homogeneous Klein-Gordon equation except the zero solution. The propagator in region I is given by:

$$
\frac{e^{i k \chi}}{\sinh \chi}, \quad \text { where } \quad \chi=D\left(x, x_{0}\right) \text {, }
$$

with $D(p, q)$ the distance in the optical metric between $p$ and $q$. Thus as $x_{0}$ goes to conformal infinity, the propagator falls off like $e^{-\chi}$. In terms of the proper distance of the pole of the propagator from the horizon $\delta$, we find that the propagator vanishes like $\delta^{1}$. This is in agreement with Teitelboim [14]. We will verify this analysis below by showing that for the $k=0$ mode we may solve the problem exactly throughout the exterior.

\subsubsection{Example - Proca equation}

The generalisation of Maxwell's equations for electromagnetism to the case where the photon is not taken to be massless is given by the Proca equations. In terms of a one-form $A$ the vacuum equations may be written:

$$
\star d \star d A+m^{2} A=0 .
$$

In this case, we may quickly estimate the rate at which the information is lost as a charged particle falls quasi-statically into a (Schwarzschild or Reissner-Nordström) back hole if the electromagnetic field is mediated by a massive vector boson. We make the ansatz:

$$
A=\frac{\psi(\boldsymbol{x})}{H(\boldsymbol{x})} d t
$$

and find that for a point charge at $x_{0}$ the function $\psi$ should satisfy:

$$
\frac{1}{H^{5}} \Delta_{h} \psi-m^{2} \frac{\Omega^{2}}{H} \psi=\frac{1}{H^{6}} \delta_{h}\left(x, x_{0}\right),
$$

where $h, H$ and $\Omega$ are as in the last section. This is once again of the form conjectured above and for $m \neq 0$ there are no solutions to the vacuum equations regular throughout the exterior of the black hole. The $m=0$ case corresponds to a Maxwell field and is treated below, however we expect a significant difference as for this case the equations are gauge invariant.

The propagator in region $I$ is given by:

$$
\frac{1}{e^{2 \chi}-1}, \quad \text { where } \quad \chi=D\left(x, x_{0}\right),
$$


where $D(p, q)$ is as above. As the pole recedes to infinity the propagator decays like $e^{-2 \chi}$ corresponding to a fall off as the square of the proper distance to the horizon, $\delta^{2}$. This decay at twice the rate of the massive scalar boson case is also in agreement with Teitelboim.

\subsubsection{Example - Forces from Neutrino pair exchange}

As noted above there is a force in flat space between leptons mediated by neutrinos which might in principle be used to measure the lepton number of a black hole. As a black hole should not have a measurable lepton number associated with it, we will now consider the problem of neutrino mediated forces in the vicinity of an event horizon. We expect such forces to vanish as a lepton approaches the horizon. We will require the following short Lemma which may be proven by considering the behaviour of the spin connection under conformal transformations.

Lemma 4.1. Suppose $\tilde{g}=\Omega^{2} g$ are two conformally related $n$-dimensional metrics and $\tilde{\psi}=\Omega^{-(n-1) / 2} \psi$ is a Dirac spinor, then

$$
\widetilde{D} \tilde{\psi}=\Omega^{-(n+1) / 2} \not D \psi
$$

Thus solutions of Dirac's equation for $g$ may be rescaled to solutions for $\tilde{g}$. Also

$$
\int_{\sigma} d \tilde{\sigma} \overline{\tilde{\phi}} \tilde{\psi}=\int_{\sigma}\left(d \sigma \Omega^{n-1}\right)\left(\Omega^{\frac{1-n}{2}}\right)^{2} \bar{\phi} \psi=\int_{\sigma} d \sigma \bar{\phi} \psi,
$$

so that orthonormality is preserved. It is not true however that if we start with a complete set then after rescaling we have a complete set. We will now restrict to the case where $d=4$ and the conformal transformation depends only on the spatial coordinates $X$ so that the metric remains static.

By considering how the equation (4.35) transforms under such a conformal transformation then assuming the rescaled solutions to Dirac's equation are complete we may calculate the interaction potential for the neutrino mediated force. Suppose that $V\left(X, X^{\prime}\right)$ represents the potential at $X$, due to an electron at $X^{\prime}$, with the metric $g$. Then the potential $\tilde{V}\left(X, X^{\prime}\right)$ for the metric $\tilde{g}$ is given by:

$$
\tilde{V}\left(X, X^{\prime}\right)=\Omega^{-3}(X) \Omega^{-2}\left(X^{\prime}\right) V\left(X, X^{\prime}\right) .
$$

There is clearly an asymmetry between the source and the test particle, however this is due the the redshift effect which means that an energy measured at different spatial points will vary.

As an example, we may consider conformally rescaling $\mathbb{R}_{t} \times \mathbb{H}^{3}$ to a metric on a static patch of the de Sitter space. In this case we pick an arbitrary point $X^{0}$ and we may write the de Sitter metric as

$$
\tilde{g}=\frac{1}{\left(-X \cdot X^{0}\right)^{2}} g
$$

where $g$ is the metric on $\mathbb{R}_{t} \times \mathbb{H}^{3}$. Suppose we take a patch with the observer at the origin, then the potential measured by this observer due to an electron at $X^{\prime}$ is given by:

$$
V(\chi)=\frac{G_{W}^{2} \cosh ^{2} \chi}{8 \pi^{3} \chi^{2} \sinh ^{4} \chi}\left(\chi \cosh \chi+\sinh \chi-\chi^{2} \text { Shi } \chi\right),
$$


with $\cosh \chi=-X \cdot X^{\prime}$. As the electron approaches the horizon, $\chi \rightarrow \infty$ and the potential is extinguished like $e^{-\chi} / \chi^{3}$, thus demonstrating the no-hair property of the de Sitter cosmological horizon for neutrino mediated forces.

Unfortunately this method does not work completely for the Schwarzschild event horizon because the conformally rescaled solutions of the Dirac equation do not form a complete basis, essentially because neutrinos may start either at spatial infinity or at the horizon, this point is made by Teitelboim and Hartle [14, 23. Accordingly, we do not reproduce precisely the extinction rate of Hartle, who finds the potential vanishes like $e^{-\chi} / \chi$, but we do find the correct exponential rate.

\subsection{Exact Calculations for Schwarzschild and Reissner-Nordström}

\subsubsection{Electric Charge}

We would like to find the field due to a static electric charge in the Schwarzschild or ReissnerNordström background. In order to do this, we make use of the fact that Maxwell's equations in vacuo:

$$
d F=0, \quad d \star_{g} F=0,
$$

are conformally invariant in 4 space-time dimensions. Thus if we can find the field due to a point particle with respect to the optical metric $g_{\text {opt. }}$ then we know the field with respect to the physical metric. The reason for this is that in 4 spacetime dimensions, for any 2 -form $\omega$ with conformal weight 0 , we have:

$$
\star_{\Omega^{2} g} \omega=\star_{g} \omega
$$

Thus the Maxwell action

$$
S=\int_{\mathcal{M}} F \wedge \star F
$$

does not change under a conformal transformation. It is also helpful to note at this stage that the charge contained inside a 2-surface $\Sigma$ :

$$
Q_{\Sigma}=\int_{\Sigma} \iota^{*}(\star F)
$$

is conformally invariant, where $\iota: \Sigma \rightarrow \mathcal{M}$ is the inclusion map and $\star$ may refer to any representative of the conformal class of $g$, by (4.81).

In order to solve the first Maxwell equation, we as usual introduce a one-form potential $A$ and make a static ansatz:

$$
F=d A, \quad A=\Phi(\boldsymbol{x}) d t .
$$

The reason for this static ansatz is primarily the fact that the resulting equations are analytically tractable. It will give a good approximation to the field of a freely falling particle, provided the particle is not moving very quickly. Alternatively, one may imagine a thought experiment where a charge is lowered from infinity towards the black hole horizon and measurements of the fields are made as the charge approaches the black hole.

The second Maxwell equation becomes the familiar Laplace equation for $\Phi$ with respect to the optical metric:

$$
d \star_{h_{\text {opt. }}} d \Phi=0
$$


We will make use of the fact noted above that

$$
h_{\text {opt. }}=H^{4} h,
$$

where $h$ is the hyperbolic metric of $\mathbb{H}^{3}$ with radius 1 and $H$ is harmonic on $\mathbb{H}^{3}$. We are therefore able to relate the Laplacian of $h_{\text {opt. }}$ to the Laplacian of $h$. We will require the following lemma:

Lemma 4.2. Suppose $h_{1}$ and $h_{2}$ are two three dimensional metrics with related Laplace operators ${ }^{1} \Delta$ and ${ }^{2} \Delta$. Further suppose that they are conformally related:

$$
h_{1}=H^{4} h_{2} \text {. }
$$

Then if $\phi=H^{-1} \Psi$ the following relation holds:

$$
{ }^{1} \Delta \phi={\frac{1}{H^{5}}}^{2} \Delta \Psi-{\frac{\Psi}{H^{6}}}^{2} \Delta H .
$$

Proof. Use the standard formula $\Delta=\frac{1}{\sqrt{g}} \frac{\partial}{\partial x^{i}} \sqrt{g} g^{i j} \frac{\partial}{\partial x^{j}}$ and make the substitutions above, then collect terms to find (4.88).

We wish to find the Green's function for the Laplacian on $\tilde{h}=h_{\mathrm{opt}}$. This satisfies the following equation:

$$
\widetilde{\Delta} \widetilde{G}\left(x, x_{0}\right)=\delta_{(\tilde{h})}\left(x, x_{0}\right),
$$

Where $\widetilde{\Delta}$ is taken to act on the $x$ coordinates. The Dirac delta function is defined by the requirement:

$$
\int_{\mathcal{U}} \delta_{(g)}\left(x, x_{0}\right) d \operatorname{vol}_{\mathrm{g}}= \begin{cases}1 & \text { if } x_{0} \in \mathcal{U} \\ 0 & \text { if } x_{0} \notin \mathcal{U}\end{cases}
$$

for an open subset $\mathcal{U} \subset \mathcal{M}$. Applying the above lemma and making use of the fact that $\Delta_{h} H=0$ we have that

$$
\widetilde{\Delta} \phi=\frac{1}{H^{5}} \Delta_{h} \Psi
$$

with $\phi$ and $\Psi$ related as above. Inserting this into the definition of the Green's function we have $\widetilde{G}\left(x, x_{0}\right)=H(x)^{-1} G\left(x, x_{0}\right)$, where $G$ obeys:

$$
\Delta_{h} G\left(x, x_{0}\right)=H(x)^{5} \delta_{(\tilde{h})}\left(x, x_{0}\right)=H\left(x_{0}\right)^{-1} \delta_{(h)}\left(x, x_{0}\right),
$$

where in this last step we use properties of the Dirac delta function. It would appear that we have simply replaced one Green's function problem with another, however the great advantage is that we now seek Green's functions on $\mathbb{H}^{3}$ which is maximally symmetric so if we can find the Green's function for $x_{0}=0$ we can generate all Green's functions by $S O(3,1)$ transformations.

Suppose $V_{O}(x)$ satisfies

$$
\Delta_{h} V_{O}(x)=\delta_{(h)}(x, O), \quad V_{O} \rightarrow \frac{1}{4 \pi} \quad \text { as } \quad D(x, O) \rightarrow \infty
$$


where $O$ is a fixed point in $\mathbb{H}^{3}$ and $D(x, O)$ is the hyperbolic distance from $x$ to $O$. In Beltrami coordinates $V_{O}(\boldsymbol{x})=(4 \pi|\boldsymbol{x}|)^{-1}$. By $S O(3,1)$ invariance, for any other point $x_{0}$ we can find a isometry $T: \mathbb{H}^{3} \rightarrow \mathbb{H}^{3}$ which satisfies

$$
T_{x_{0}}^{*} h=h, \quad T_{x_{0}}\left(x_{0}\right)=O .
$$

We then define

$$
V_{x_{0}}=T_{x_{0}}^{*} V_{O}, \quad \text { i.e. } \quad V_{x_{0}}(x)=V_{O}\left(T_{x_{0}}(x)\right) .
$$

The map $T$ is not uniquely defined, but since $V_{O}$ is spherically symmetric any two maps $T$ satisfying (4.94) give the same function $V_{x_{0}}$. This new function satisfies

$$
\Delta_{h} V_{x_{0}}(x)=\delta_{(h)}\left(x, x_{0}\right) .
$$

Putting together (4.92) and (4.96) we find that the Green's function for the Laplacian of $h_{\text {opt. }}$ has the form

$$
\widetilde{G}\left(x, x_{0}\right)=\frac{1}{H(x) H\left(x_{0}\right)}\left(V_{O}\left(T_{x_{0}}(x)\right)+A\right)+B .
$$

We have now to specify boundary conditions. The constant $B$ is unphysical, and if we choose $B=0$, the potential will vanish at the asymptotically flat end. The constant $A$ arises because if $V_{x_{0}}$ satisfies (4.96) then so does $V_{x_{0}}+A$. This constant gives rise to a non-trivial field which corresponds to the black hole carrying a (linearised) charge. Transforming to Eddington-Finkelstein coordinates shows that the function $\widetilde{G}$ is regular at the horizon for all values of $A$, so we must look elsewhere for our final boundary condition. This comes from the fact that Gauss' law should be satisfied. If one considers a surface $\Sigma$ which encloses the black hole, but not the point $x_{0}$ then $Q_{\Sigma}$ should vanish. Enforcing this condition fixes $A$. In the case where the harmonic function $H$ takes the Reissner-Nordström form:

$$
\sqrt{\mu} H(x)=4 \pi \mu V_{O}(x)+m
$$

Gauss' law requires $A=\frac{m}{4 \pi \mu}$ and we finally have:

$$
\widetilde{G}\left(x, x_{0}\right)=\frac{1}{H(x) H\left(x_{0}\right)}\left(V_{O}\left(T_{x_{0}}(x)\right)+\frac{m}{4 \pi \mu}\right) .
$$

This construction is valid for both Schwarzschild and Reissner-Nordström and gives the linear perturbation to the electromagnetic field due to a static point charge located in the spacetime. The potential has been found in terms of geometric objects of hyperbolic space, and so is valid for any coordinate system on $\mathbb{H}^{3}$.

We can see from this equation how the information associated with the precise location of the charged particle is lost as it is lowered towards the black hole. The only term which is not spherically symmetric about $O$ in (4.99) is the $V_{O}$ term. As the point $x_{0}$ recedes from $O$ towards the black hole horizon which is at the conformal infinity of $\mathbb{H}^{3}$, this term approaches a constant exponentially quickly in $D\left(x_{0}, O\right)$. The potential tends to the spherically symmetric field associated with the black hole carrying a charge and deviations from this field fall exponentially with $D\left(x_{0}, O\right)$.

We plot below the isopotentials for a point charge in Schwarzschild, taking isotropic coordinates so that the spatial sections are conformally flat and the lines of force are normal to the isopotentials. Isotropic coordinates for Schwarzschild correspond to Poincaré coordinates on the hyperbolic space from which the optical metric is constructed. The black region in the plots corresponds to the interior of the black hole event horizon and we have returned the asymptotically flat end to infinity. 

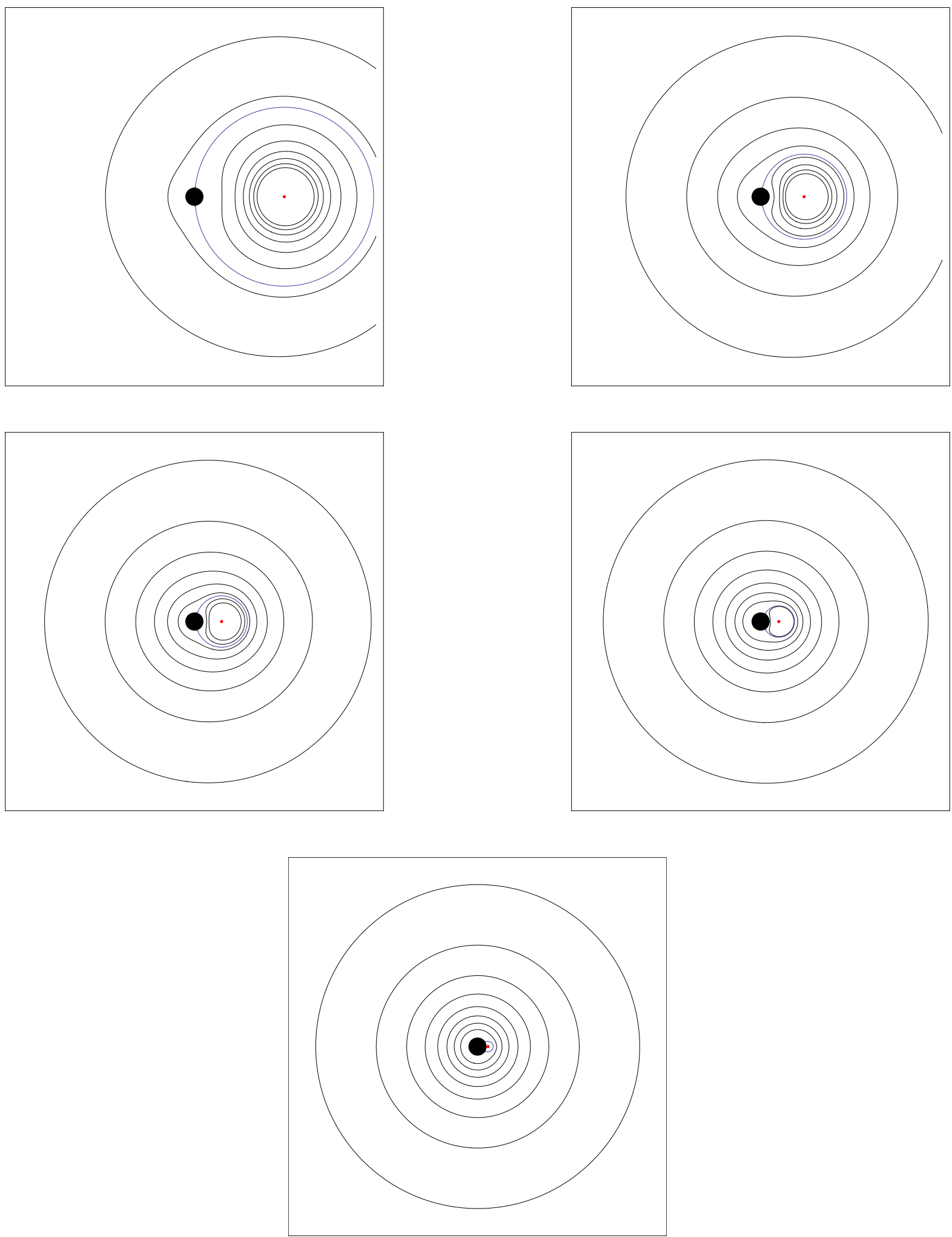

Figure 3: Plots showing the equipotentials as a point charge is lowered into a Schwarzschild black hole in isotropic coordinates. The horizon is located at the boundary of the black disc and the point charge is red. The blue contour is the equipotential of the horizon. 


\subsubsection{Scalar Charge}

We will now show how to treat exactly a static massless scalar field in the Schwarzschild or ReissnerNordström backgrounds. We will once again make use of the optical metric and the relationship between this metric and the hyperbolic metric. The main result we shall require is summarised as

Lemma 4.3. If $g$ is a scalar flat static metric of the form:

$$
g=\Omega^{2} g_{\text {opt. }}=\Omega^{2}\left(-d t^{2}+H^{4} h\right),
$$

with $h$ the metric on $\mathbb{H}^{3}$ with radius 1 and $H$ harmonic on $\mathbb{H}^{3}$ then $\Omega$ must satisfy

$$
\Delta_{h}(\Omega H)+\Omega H=0 .
$$

Further, if $\psi=\Omega^{-1} H^{-1} \Phi$, then

$$
\square_{g} \psi=\Omega^{-3}\left[-\frac{1}{H} \frac{\partial^{2} \Phi}{\partial t^{2}}+\frac{1}{H^{5}}\left(\Delta_{h} \Phi+\Phi\right)\right] .
$$

Proof. This follows from standard identities for conformal transformations.

We would like to calculate the field $G\left(x, x_{0}\right)$ at $x$ due to a unit static scalar charge at $x_{0}$. For a general moving point charge, $G$ satisfies:

$$
\square_{g} G\left(x, x^{\prime}(\tau)\right)=\int_{-\infty}^{\infty} \delta_{(g)}\left(x, x^{\prime}(\tau)\right) d \tau,
$$

where $\tau$ is the proper time along the worldline $x^{\prime}(\tau)$ of the charged particle. Assuming that this particle is static, we find using the Lemma above that $G\left(x, x_{0}\right)=\Omega^{-1}(x) H^{-1}(x) \Phi\left(x, x_{0}\right)$ where $\Phi$ satisfies

$$
\Delta_{h} \Phi\left(x, x_{0}\right)+\Phi\left(x, x_{0}\right)=H^{-1}\left(x_{0}\right) \delta_{(h)}\left(x, x_{0}\right) .
$$

It is convenient once again to make use of the $S O(3,1)$ invariance of hyperbolic space in order to solve this equation. We first seek solutions to the simpler equation

$$
\Delta_{h} \Phi_{O}(x)+\Phi_{O}(x)=\delta_{(h)}(x, O)
$$

subject to the condition that $\Phi$ and $d \Phi$ are bounded in the metric induced by $h$ as $D(x, O) \rightarrow \infty$. One finds that the solution is related to the metric functions for Reissner-Nordström according to:

$$
\Phi_{O}(x)=\frac{1}{4 \pi \sqrt{\mu}} \Omega(x) H(x) .
$$

Unlike in the scalar charged case, there is no arbitrary constant. Using the identities above, we find that the field due to a static unit scalar charge at a point $x_{0}$ is given by:

$$
G\left(x, x_{0}\right)=\frac{\Phi_{O}\left(T_{x_{0}}(x)\right)}{H(x) H\left(x_{0}\right) \Omega(x)},
$$

where $T_{0}$ is defined as in the previous section.

We may once again consider the behaviour of $G\left(x, x_{0}\right)$ as the scalar charge approaches the horizon. We find that $G$ and its derivatives fall off like $e^{-D\left(x_{0}, O\right)}$ as $D\left(x_{0}, O\right) \rightarrow \infty$. Unlike the case of an electric charge, there is no residual monopole term, so the black hole does not become charged. Thus, we see precisely how massless scalar hair is shed as a point scalar charge is lowered into a black hole and it it as predicted by our approximate argument given above. 


\section{Conclusion}

We have seen how it is possible to make use of the universal asymptotics of the optical metric near a Killing horizon to study physical problems in this region. We have presented a method of studying null geodesics based on the Gauss-Bonnet theorem which directly links the negative curvature of the optical geometry to physical lensing scenarios. We have re-derived classic results about the loss of 'hair' as objects fall into a black hole in a simplified manner and by making use of the universality of the near horizon optical metric, extended these results to apply beyond the Schwarzschild case where they were first investigated.

\section{A Integration on $\mathbb{C P}^{1}$}

In section 4.1 we found that the space of solutions to Dirac's equation on $\mathbb{R}_{t} \times \mathbb{H}^{3}$ could be identified with $\mathbb{R}_{+} \times \mathbb{C P}^{1}$ where the $\mathbb{C P}^{1}$ arose by identifying Weyl spinors which were complex multiples of one another. In subsequent calculations it was necessary to integrate over this space of solutions in a Lorentz invariant fashion. The aim of this appendix is to explain how this is possible.

There are two key observations to be made. Firstly it should be noted that the space of Weyl spinors caries a natural 2-form defined by:

$$
\mu[\chi]=2 i \epsilon_{\alpha \beta} \chi^{\alpha} d \chi^{\beta} \wedge \epsilon_{\dot{\alpha} \dot{\beta}} \bar{\chi}^{\dot{\alpha}} d \bar{\chi}^{\dot{\beta}} .
$$

This is Lorentz invariant by construction.

Secondly we may represent $\mathbb{C P}^{1}$ as a smooth 2 -dimensional surface in $\mathbb{C}^{2}, \Sigma$ where we assume that for almost every $[\chi] \in \mathbb{C P}^{1}$ there is exactly one point $\tilde{\chi} \in \Sigma$ such that $[\chi]=[\tilde{\chi}]$. Since we are interested in integrating over $\mathbb{C P}^{1}$ it doesn't matter if this fails to be true for some set of measure zero. Suppose now that we chose a different surface $\Sigma^{\prime}$. In order that this fulfils the requirements to represent $\mathbb{C P}^{1}$ there must exist some smooth function $\lambda: \mathbb{C}^{2} \rightarrow \mathbb{C}$ such that for almost every point $\chi \in \Sigma, \lambda(\chi) \chi \in \Sigma^{\prime}$. In other words we may, by extending the domain of $\phi$ if necessary define a local diffeomorphism

$$
\begin{aligned}
\phi: U \subset \mathbb{C}^{2} & \rightarrow U^{\prime} \subset \mathbb{C}^{2} \\
\chi & \mapsto \lambda(\chi) \chi
\end{aligned}
$$

such that $\phi(\Sigma)=\Sigma^{\prime}$ up to a set of measure zero. One may verify that

$$
\phi^{*} \mu=|\lambda|^{4} \mu \text {. }
$$

Thus if we have a function $f: \mathbb{C}^{2} \rightarrow \mathbb{C}$ which is a scalar under Lorentz transformations and which satisfies $f(\lambda \chi)=|\lambda|^{-4} f(\chi)$ then the integral

$$
\int_{\Sigma} f \mu
$$

is independent of which surface in $\mathbb{C}^{2}$ we use to represent $\mathbb{C P}^{1}$. Suppose now that $f=f\left(\chi, X^{i}\right)$ where $X^{i}$ are some vectors in $\mathbb{E}^{3,1}$ and such that

$$
f\left(\rho_{\Lambda}^{s} \chi, \rho_{\Lambda}^{v} X^{i}\right)=f\left(\chi, X^{i}\right)
$$


where $\rho^{s}, \rho^{v}$ are the spinor and vector representations of the Lorentz transformation $\Lambda$ respectively. If we pick a surface $\Sigma$ which represents $\mathbb{C P}^{1}$, we may define a function

$$
I\left(X^{i}\right)=\int_{\Sigma} f\left(\chi, X^{i}\right) \mu=\int_{\Sigma} f\left(\rho_{\Lambda}^{s} \chi, \rho_{\Lambda}^{v} X^{i}\right) \mu .
$$

If $\phi_{\Lambda}$ is the function on $\mathbb{C}^{2}$ defined by left multiplication by $\rho_{\Lambda}^{s}$ then we may use the Lorentz invariance of the measure $\mu$ to write

$$
\begin{aligned}
I\left(X^{i}\right) & =\int_{\Sigma} \phi_{\Lambda}^{*}\left(f\left(\chi, \rho_{\Lambda}^{v} X^{i}\right) \mu\right)=\int_{\phi_{\Lambda}(\Sigma)} f\left(\chi, \rho_{\Lambda}^{v} X^{i}\right) \mu \\
& =\int_{\Sigma} f\left(\chi, \rho_{\Lambda}^{v} X^{i}\right) \mu=I\left(\rho_{\Lambda}^{v} X^{i}\right)
\end{aligned}
$$

where we have made use of the Lorentz invariance of $f$ and $\mu$, together with the independence of the integral on the choice of representative of $\mathbb{C P}^{1}$. Thus the integral is a Lorentz scalar a fact which we make use of in section 4.1.4 to calculate the integral (4.53)

As an example, we may take $\Sigma=\left\{(1, z)^{t} /\left(1+|z|^{2}\right)^{1 / 2}: z \in \mathbb{C}\right\}$ which covers all of $\mathbb{C P}^{1}$ except one point. We find then that

$$
\left.\mu\right|_{\Sigma}=\frac{2 i}{\left(1+|z|^{2}\right)^{2}} d z \wedge d \bar{z}
$$

the standard measure on the sphere under stereographic projection. We use this fact in calculating the neutrino mediated force between electrons.

\section{References}

[1] I. Sachs and S. N. Solodukhin, "Horizon holography," Phys. Rev. D 64 (2001) 124023 arXiv:hep-th/0107173.

[2] Z. Haba, "Green functions and dimensional reduction of quantum fields on product manifolds," Class. Quant. Grav. 25 (2008) 075005 [arXiv:0709.3227 [hep-th]].

[3] G. W. Gibbons and S. N. Solodukhin, "The Geometry of Large Causal Diamonds and the No Hair Property of Asymptotically de-Sitter Spacetimes," Phys. Lett. B 652 (2007) 103 arXiv:0706.0603 [hep-th]].

[4] G. W. Gibbons, "No glory in cosmic string theory," Phys. Lett. B 308 (1993) 237.

[5] K. S. . Thorne, R. H. . Price and D. A. . Macdonald, "Blach Holes: the membrane paradigm," New Haven, USA: Yale Univ. Pr. (1986)

[6] S. R. Das, G. W. Gibbons and S. D. Mathur, Phys. Rev. Lett. 78 (1997) 417 arXiv:hep-th/9609052.

[7] G. Policastro, D. T. Son and A. O. Starinets, Phys. Rev. Lett. 87 (2001) 081601 arXiv:hep-th/0104066. 
[8] E. T. Copson, Proc. R. Soc. A 118 (1928) 184.

[9] B. Linet, "Electrostatics and magnetostatics in the Schwarzschild metric," J. Phys. A 9 (1976) 1081.

[10] B. Leaute and B. Linet, "Electrostatics in a Reissner-Nordstrom space-time," Phys. Lett. A 58 (1976) 5.

[11] M. A. Abramowicz, I. Bengtsson, V. Karas and K. Rosquist, "Poincare ball embeddings of the optical geometry, Class. Quant. Grav. 19 (2002) 3963 [arXiv:gr-qc/0206027].

[12] G. W. Gibbons and M. C. Werner, "Applications of the Gauss-Bonnet theorem to gravitational lensing," arXiv:0807.0854 [gr-qc].

[13] O Lodge Nature 104 (1919) 354

[14] C. Teitelboim, "Nonmeasurability of the quantum numbers of a black hole," Phys. Rev. D 5 (1972) 2941.

[15] C. G. . Callan and F. Wilczek, "Infrared behaviour at negative curvature," Nucl. Phys. B 340 (1990) 366.

[16] M. Atiyah and P. Sutcliffe, "Skyrmions, instantons, mass and curvature," Phys. Lett. B 605, 106 (2005) arXiv:hep-th/0411052.

[17] G. Cognola and L. Vanzo, "Bose-Einstein condensation of scalar fields on hyperbolic manifolds," Phys. Rev. D 47, 4575 (1993) arXiv:hep-th/9210003.

[18] Dennis DeTurck and Herman Gluck, J. Math. Phys. 49, 023504 (2008), "Electrodynamics and the Gauss linking integral on the 3-sphere and in hyperbolic 3-space," DOI:10.1063/1.2827467

[19] P. A. M. Dirac, "The electron wave equation in de-Sitter space" Ann. Math. 36 (1935) 657

[20] D. V. Paramonov, N. N. Paramonova and N. S. Shavokhina, "The Dirac equation in the Lobachevsky space time," JINR-E2-2000-79;

[21] U. Moschella and R. Schaeffer, "Quantum Theory on Lobatchevski Spaces," Class. Quant. Grav. 24 (2007) 3571 arXiv:0709.2795 [hep-th]].

[22] G. Feinberg and J. Sucher, Phys. Rev. 166, 1638 (1968)

[23] J. B. Hartle, "Can A Schwarzschild Black Hole Exert Long Range Neutrino Forces?" In *J $R$ Klauder, Magic Without Magic*, San Francisco 1972, 259-275

[24] E. V. Gorbar, "Dynamical symmetry breaking in spaces with constant negative curvature," Phys. Rev. D 61, 024013 (2000) arXiv:hep-th/9904180. 